\title{
Processes of Direct Laser Writing 3D Nano-Lithography
}

\author{
Simonas Varapnickas and Mangirdas Malinauskas
}

\begin{abstract}
Direct laser writing three-dimensional nano-lithography is an established technique for manufacturing functional 3D micro- and nano-objects via non-linear absorption induced polymerization process. In this Chapter an underlying physical mechanisms taking place during nano-confined polymerization reaction, induced by tightly focused ultra-short laser pulses, are reviewed and discussed. The special attention is paid on the effects that directly impact structuring resolution and minimum achievable feature size. Analysis of possible photo-initiation mechanisms as contributing multi-photon absorption and avalanche ionization in pre-polymers under diverse exposure conditions (wavelength, pulse duration) is presented. Feasible structuring of pure (non-photosensitized) and functional nanoparticles doped polymer precursors is justified and benefits of such materials/structures for microoptics, photonics and cell scaffolds are highlighted. The influence of temperature effects (induced by writing process itself or determined by ambient conditions) on polymerization process, observed in different pre-polymers under diverse exposure regimes is outlined. The further adjustment of the structuring resolution is possible via precise control of light polarization and diffusion assisted radical quenching. The work is concluded with a brief outlook on future challenges and perspectives related to refinement of 3D ultra-fast laser lithography fabrication process in the means of application of diverse post-processing methods and research into novel photo-curable materials including inorganic ones.
\end{abstract}

Key words: 3D nano-lithography, 3D laser lithography, direct laser writing, nanopolymerization, cross-linking, multi-photon absorption, avalanche ionization, temperature effects

Simonas Varapnickas

Laser Research Center, Faculty of Physics, Vilnius University, Sauletekio Ave. 10, LT-10223, Vilnius, Lithuania e-mail: simonas.varapnickas@ ff.vu.lt

Mangirdas Malinauskas

Laser Research Center, Faculty of Physics, Vilnius University, Saulėtekio Ave. 10, LT-10223, Vilnius, Lithuania e-mail: mangirdas.malinauskas@ff.vu.lt 


\section{Introduction}

Fabrication technique, enabling creation of true three-dimensional (3D) polymer micro-structures using tightly focused ultra-fast laser beam, was first introduced at the end of last millennium (Maruo et al., 1997). The method was initially denominated as two-photon-absorbed (TPA) photo-polymerization, as the solidification of acrylate resin occurred due to a radical polymerization under exposition by a near-infrared (NIR) femtosecond (fs) pulses (which are off-resonance for both monomers and photo-initiator molecules). During the two decades of intense research and development in the field, the technology was adopted for micro-printing out of various polymer precursors, including but not limited to, acrylate- and epoxybased resins, hydrogels or hybrid organic-inorganic pre-polymers. Consequently, different application fields emerge from the wide selection of physical and chemical properties of printable materials. For example, low shrinkage and optically clear polymers are widely used for production of micro-/nano-photonic components (or entire devices) (Dietrich et al., 2018), while the low cytotoxicity, flexibility and bio-compatibility of hydrogels is beneficial for scaffolds for tissue regeneration (Torgersen et al., 2012). The scope of possible applications gets even wider if composite materials are being considered. Dozens of successful attempts to combine two or more polymers with different properties and (or) to dope pre-polymer with functional nanoparticles (NPs), i.e. high refractive index, magnetic, plasmonic or luminescent, were reported during the recent years (Sekkat and Kawata, 2013). Such enhanced functionality of 3D micro-/nano-structures certainly will be utilized to create conceptually new micro-nano-sensors in the very near future.

Besides the variety of investigated materials, 3D nano-polymerization process was demonstrated under completely different laser exposure conditions. Not only the Ti:Sapphire oscillator, which was the primary choice for TPA photo-polymerization, but also the amplified laser systems operating at different pulse duration (from hundreds of femtoseconds to nanoseconds (Stankevičius et al., 2017)) and even continuous-wave (CW) lasers (Do et al., 2013) successfully utilized for 3D nanoprinting. Evidently, this suggests pure TPA not to be the only possible polymerization initiation pathway, but rather one of the several contributing mechanisms. Likely, this variety of different experimental conditions caused the diversity of terms used by the different authors, entitling virtually the same technology, i.e. TPA/TPP (sometimes abbreviated as 2PA/2PP) (nano)polymerization, MPA or MPP multi-photon(absorbed) (nano)polymerization, or simply non-linear 3D laser (nano)lithography (3DLL). Commonly, the term direct laser writing (DLW) lithography is used interchangeably to emphasize the technological implementation of the process rather than the underlying physical mechanism. ${ }^{1}$

Despite the ambiguous theoretical background, the technological implementation of 3DLL already reached its maturity. Fabrication of micro-nano-structures

1 Other abbreviations sometimes appear in scientific literature. In this work terms TPA/MPA (nano)polymerization will be used to highlight the initiation mechanism and DLW (nano)lithography or $3 D L L$ to denominate a technological process. 
with minimal feature sizes of sub- two hundred nanometers, is being achieved repeatedly in a number of research laboratories. Furthermore, the great advances were achieved in terms of 3D structuring accuracy and speed, thanks to commercially available advanced opto-electrical/mechanical solutions, i.e. long travel (up to tens of centimeters) linear nano-positioners and fast yet precise galvanometer scanners. Sophisticated synchronization algorithms of above-mentioned modules enable so called meso-scale manufacturing - fabricating structures that reach millimeters in size but still retain $\mu \mathrm{m}$-scale features (Jonušauskas et al., 2018). Generally talking, DLW nano-lithography systems are no longer the tool for research laboratories only. The turn-key workstations, pre-adjusted for specific manufacturing needs and compatible with industrial environments are already available in the market, accelerating lab-to-fab transfer of the technology (Malinauskas et al., 2016).

Nevertheless, many authors would agree that underlying physical mechanisms of nano-polymerization reaction induced by tightly focused laser pulses remain uncertain. No generalized model can unequivocally and simultaneously explain the influence of pulse duration, polarization, pulse repetition rate versus writing velocity for nano-polymerization kinetics. In this Chapter we review and discuss an underlying physical phenomena, backed by the empirical observations (documented by our as well as other research groups). As the key exception of DLW lithography is ability to produce virtually free-form, sub-diffraction-limited resolution of printed structures, the special attention will be paid on mechanisms and effects that have a direct impact on structuring resolution and a minimum feature size achievable.

The structure of the Chapter is organized as follows: after introduction present in Section 1 a short presentation of the fundamentals of DLW nano-lithography is given in Section 2. In Section 3 we present an overview of possible photoinitiation mechanisms, the contribution of multi-photon absorption versus avalanche ionization in pre-polymers. Section 4 is devoted for 3D polymerization in pure (non-photosensitized) and functional nanoparticles doped polymer precursors. The temperature effects on polymerization process and polymerized structures is discussed in Section 5. Section 6 is dedicated for light polarization induced effects. Diffusion assisted radical quenching phenomenon is described in Section 7. The Chapter is concluded by Section 8 , where a brief outlook on future challenges and perspectives related to furthering of 3DLL fabrication process is suggested.

\section{Fundamentals of Direct Laser Writing 3D Nano-Lithography}

In 3DLL process (which is schematically illustrated in Fig. 1), a polymer structure is being formed via non-linear absorption of spatio-temporally localized light inside pre-polymer material, deposited on a substrate (i.e. glass). Sufficient light concentration is achieved when ultra-short laser pulses (typically of sub-ps duration) are tightly focused into photo-curable polymers (Fig. 1 (a)) through a high numerical aperture $(N A)$ objective $(N A>0.5$, usually $1.25-1.4)$. The photosensitive resins used are transparent in the visible to NIR spectral range $(\lambda>400 \mathrm{~nm})$, thus, NIR 
laser pulses can be focused into the volume of the resin. This makes 3DLL process conceptually different from the linear absorption (single photon) lithography, which is a planar process restricted to the surface (or layer thickness) of the resin. If the photon density exceeds a certain threshold value (detailed in Sect. 3), two-photon absorption occurs within the focal volume, initiating the polymerization process. By setting only the very top of the laser Gaussian beam intensity over this threshold, photo-modified volumes called voxels (volumetric pixels) with dimensions smaller than the one predicted by optical diffraction can be achieved. If the laser focus is moved three-dimensionally through the volume of the resin, the polymerization process is initiated along the track of the focus, allowing the fabrication of any 3D micro-structure (Fig. 1 (b)). For the resin to undergo a phase transformation that produces features with enough structural integrity to survive the developing step, a sufficient degree of conversion (cross-linking) should be achieved.

Tightly focused beams of high quality factor $\left(M^{2} \approx 1\right)$ are used for DLW, thus, spatial profiles near the focal plane can be well approximated by a Gaussian function. The spatial bell-shaped Gaussian profile fits well the electrical field strength, $E[\mathrm{~V} / \mathrm{m}]$, energy $E_{n}[\mathrm{~J}]$, photon flux $N\left[\mathrm{~cm}^{-2} \mathrm{~s}^{-1}\right]$, fluence $F\left[\mathrm{~J} / \mathrm{cm}^{2}\right]$, irradiance $I\left[\mathrm{~J} /\left(\mathrm{s} \mathrm{cm}^{2}\right)\right]$ (intensity $\left.I=\frac{1}{2} \epsilon_{0} c n_{0}|E|^{2}\right)$, and dose $D=I \times t_{e x}\left[\mathrm{~J} / \mathrm{cm}^{2}\right]\left(t_{e x}\right.$ is the exposure time) distributions. The generic Gaussian distribution at focus of all those parameters is described by $A$ :
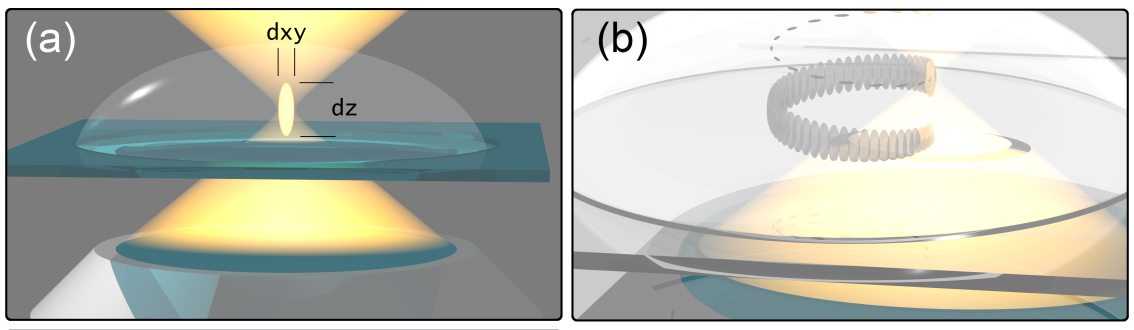

(c)

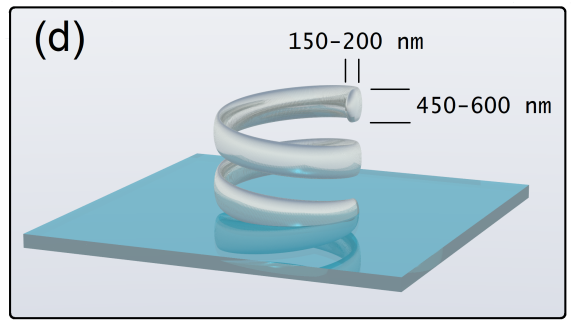

Fig. 1 The principle of direct laser writing3D nano-lithography. (a) Tightly focused laser pulses induce permanent photo-modification in the high intensity region. Shape of the focal region defined by a constant-intensity surface is ellipsoidal, with axial and lateral diameters $\mathrm{d} z$ and $\mathrm{d} x y$, respectively. For tight focusing with an objective lens $N A \sim 1.4$, the elongation ratio is $d_{z} / d_{x y} \approx 3$. (b) Continuous sample (or focal spot) translation in $3 \mathrm{D}$ space results in photo-modificated patterns. (c) Non-exposed resin is washed out during a wet chemical development and subsequent rinse procedures. (d) Solid self-standing micro-structures are retrieved. 
Processes of Direct Laser Writing 3D Nano-Lithography

$$
A(r)=A_{0} \exp \left(\frac{-2 r^{2}}{r_{0}^{2}}\right),
$$

where $r_{0}$ is the waist of the beam, $A_{0}$ is the amplitude of either $E\left(E_{n}, N, I, F, D\right)$, $r$ is the radius to the axis of a beam. For a Gaussian beam the fluence is given by $F_{0}=2 E_{0} /\left(\pi r_{0}^{2}\right)$ ). The fluence and dose are usually relevant parameters for consideration of modifications induced by the absorbed laser light energy at the focal region of a sample. When the material modification is induced by non-linear optical absorption, the intensity becomes an important parameter since the modification by an n-photon process scales as $\propto I^{n}$. In fact, a contribution of both, process nonlinearity and thresholding behavior of photo excitation as well as chemical reaction, is essential for sub-diffraction-limited structuring resolution. Thus, the minimal feature size of photo-cured structures retrieved after wet development and subsequent rinsing (Fig. 1 (c)) can be significantly smaller than that of the laser wavelength (Fig. 1 (d)). When lasers operating at near-infrared and visible wavelengths are used, reproducible and mechanically stable structures with feature size of 200-300 nm are achievable routinely. In case near-threshold exposition intensity condition is precisely maintained, photo-polymerized 3D structures with cross-sections comparable with tens of molecular diameters (corresponding to $\lambda / 25$ resolution) can be obtained (Juodkazis et al., 2005).

Different technical implementations of DLW systems might be found used for practical exploitation of 3DLL method. However, the main functional units (i.e. light sources, beam shaping and delivery units and precise motion system) are always present (see the diagram of a typical experimental setup in Fig. 2. With only a few exceptions, either fs-laser oscillators or amplified laser systems are used as irradiation

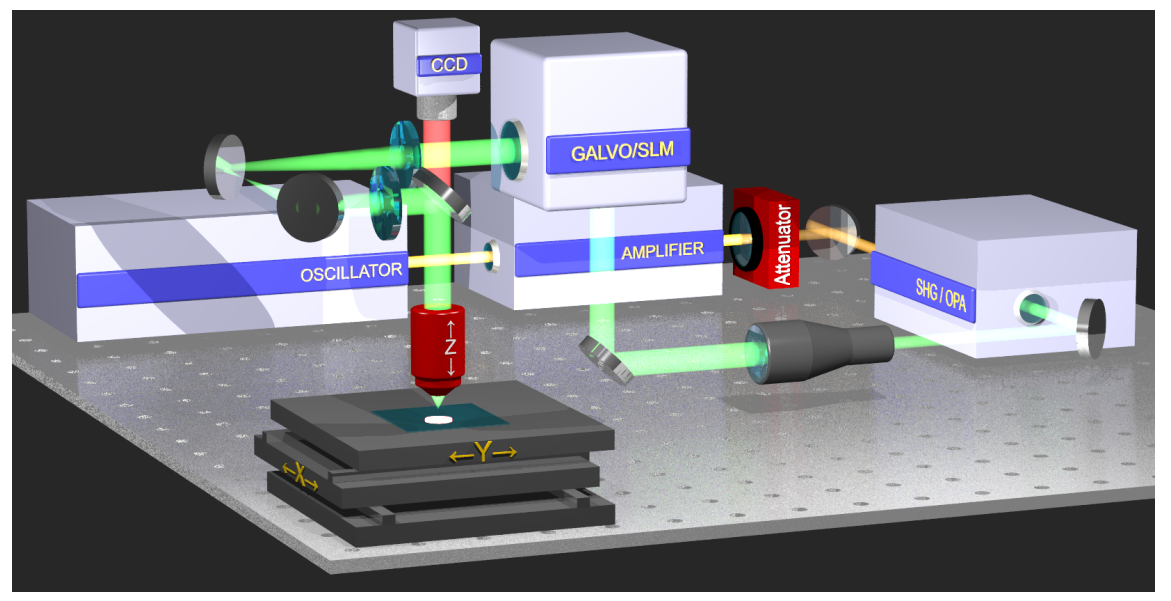

Fig. 2 Schematic illustration of typical laboratory grade DLW 3D nanopolymerization setup: 3D movable pre-polymer sample is exposed by a tightly focused fs-laser beam. Tuning of the pulsed beam parameters (energy, wavelength, diameter) is performed in beam delivery line (amplifier, SHG/OPA and GALVO/SLM units are not mandatory for the process, but increases functionality of the system). 
sources. Common parameters of lasers are in the range of: repetition rate $\sim 1 \mathrm{kHz}$ to $80 \mathrm{MHz}$, average optical power $\sim 200 \mathrm{~mW}$ to $10 \mathrm{~W}$, pulse duration from $20 \mathrm{fs}$ to $1 \mathrm{ps}$, fundamental wavelength from $800 \mathrm{~nm}$ to $1600 \mathrm{~nm}$ and higher harmonics $(400 \mathrm{~nm}$, $515 \mathrm{~nm}, 532 \mathrm{~nm}, 800 \mathrm{~nm}$, etc.).

The beam delivery unit usually incorporates precise power attenuation stage, fixed or adjustable beam expander and polarization state adjustment stage. Second harmonics generator (SHG) and/or optical parametric amplifier (OPA) are optional, but might be found advantageous as frequency doubling or parametric up-/downconversion fully suppresses a nanosecond-long pedestal of fs-pulses. This is important for laser structuring regime, in which free charges seeded by ultra-short high peak intensity pulses are further multiplied due to avalanche ionization process (refer to Sec. 3 for details). In case a long laser pulse pedestal is present, avalanche ionization extends to nanosecond scale, causing a decrease in structuring resolution. Additional beam control and shaping by spatial light modulators (SLM) or digital mirror arrays (DMD) is also possible, yet not very commonly integrated in experimental setups. As the setup can also serve as a microscope - a camera (i.e. CCD Charge Coupled Device or CMOS - Complementary Metal-Oxide-Semiconductor) can be integrated in the system to enable on-line process monitoring.

A nano-precision positioning system optimized for sample translation (or a beam deflection) in 3D space is a necessity in DLW micro/nano-structuring. Different strategies for practical implementation of 3D linear sample translation are possible. One may choose to use ultimate resolution $(\sim 1 \mathrm{~nm})$ but low speed $(\sim 100 \mu \mathrm{m} / \mathrm{s})$ and short travel $(\sim 100 \mu \mathrm{m})$ piezo stages accompanied by diverse mechanical positioners for sample repositioning, typically of long travel range and fast, but not that precise. Alternatively, a set of linear motors powered, long travel positioning stages might be utilized. The later choice compromises a minimal step resolution (down to $\sim 10 \mathrm{~nm}$ ) but dramatically increases the writing velocity (to $\mathrm{cm} / \mathrm{s}$ or even more).

Instead of sample translation, galvanometric (GALVO) or electro-optical scanners, in combination with so called $4 \mathrm{~F}$ correlator optical setup, can be used to direct the beam to the desired location. Setups of highest functionality benefits from combination of both approaches (sample motion and beam scanning). In case an advanced synchronization algorithms are applied, efficient fabrication of relatively large ( mm scale) stitch-free structures can be achieved (Jonušauskas et al., 2018).

The control of overall 3DLL systems usually is automated using custom made or commercial software for 3D DLW applications. Accordingly, 3D structures can be designed directly or imported from stereolitography CAD (STL) files.

\section{Photo-Initiation Mechanisms}

The established understanding of DLW polymerization process relies on the polymerization threshold model. High density of photons localized within small volume of photopolymer prompts multi-photon absorption by dedicated photo-initiator molecules. Upon the simultaneous absorption of two (or more) photons the photo- 
initiator is excited from its electronic singlet ground state $\left(S_{0}\right)$ to the excited singlet manifold $\left(S_{1}\right)$. From there, it relaxes to the lowest $S_{1}$ state, undergoes inter-system crossing (ISC) to the lowest triplet state, and from there forms radicals ${ }^{2}$. The density of radicals $\rho$ produced by femtosecond laser pulses via TPA can be calculated using a rate equation:

$$
\frac{\partial \rho(r, t)}{\partial t}=\left(\rho_{0}-\rho(r, t)\right) \sigma_{2} N^{2}(r, t)
$$

where $\sigma_{2}=\sigma_{2}^{a} \cdot \eta$ is the effective two-photon cross section for the generation of radicals $\left[\mathrm{cm}^{4} \mathrm{~s}\right]$, which is defined by the product of the ordinary two-photon absorption cross section, $\sigma_{2}^{a}$, and the efficiency of the initiation process, $\eta<1$; $N=$ $N(r, z, t)$ is the photon flux, and $\rho_{0}$ is the primary initiator particle density (Serbin et al., 2003). The threshold model implies, that polymerization process occurs when density of radicals, $\rho=\rho(r, z, t)$ exceeds a certain minimum concentration (threshold value) $\rho_{t h}$, which is large enough to surpass radicals quenching by oxygen, and ensures sufficient cross-linking degree for structures to survive development and rinsing stages. Assuming the light distribution to be Gaussian-shaped $(N(r, t)=$ $\left.N_{0}(t) \exp \left(-2 r^{2} / r_{0}^{2}\right)\right)$, the photon flux to be constant during the laser pulse $\left(N_{0}(t)=\right.$ $N_{0}$ ) and neglecting the losses of radicals between the laser pulses, the following equations, estimating lateral $(d x y)$ and axial $(d z)$ voxel dimensions, where $\rho \geq \rho_{t h}$ is fulfilled, can be derived:

$$
\begin{aligned}
& d x y\left(N_{0}, t\right)=r_{0} \sqrt{\ln \left(\frac{\sigma_{2} N_{0}^{2} f t \tau_{p}}{\ln \left(\frac{\rho_{0}}{\rho_{0}-\rho_{s l}}\right)}\right)} \\
& d z\left(N_{0}, t\right)=2 z_{R} \sqrt{\sqrt{\frac{\sigma_{2} N_{0}^{2} f t \tau_{p}}{\ln \left(\frac{\rho_{0}}{\rho_{0}-\rho_{s l}}\right)}-1}}
\end{aligned}
$$

where $f$ - laser pulse repetition rate, $t$ - total irradiation time, $\tau_{p}$ - laser pulse duration, $z_{R}$ - Rayleigh length (Serbin et al., 2003).

As was mentioned in Sect. 1, photo-initiation of polymerization reaction was experimentally validated applying laser pulses with duration spanning from tens of nanoseconds to tens of femtoseconds. Such variation of the pulse duration over $\sim 6$ orders of magnitude in polymerization is not compatible with the theory of the pure multi-photon absorption pathway, however, could be explained by a weighty contribution of avalanche processes in bond breaking and subsequent polymerization. This theory, introduced a decade ago, is different from the common understanding of polymerization initiation.

\footnotetext{
2 This is true for most Norish type I photo-initiators. The radicals' formation mechanism for Norish type II photo-initiators is different and more complex. See Ref. Yagci et al. (2010) for details.
} 
Estimation of Ionization Rates

The multi-photon ionization $\left(w_{m p i}\right)$ and impact/avalanche ionization $\left(w_{\text {imp }}\right)$ rates contributing to free carrier multiplication are given by (Gamaly, 2011):

$$
\begin{aligned}
& w_{m p i} \simeq \omega n_{p h}^{3 / 2}\left(\frac{\varepsilon_{o s c}}{2 \Delta E}\right)^{n_{p h}}, \\
& w_{i m p} \simeq \frac{\varepsilon_{o s c}}{\Delta E} \frac{2 \omega^{2} v_{e-p h}}{\left(v_{e-p h}^{2}+\omega^{2}\right)},
\end{aligned}
$$

where $n_{p h}=\Delta E e /(h \omega)+1$ is the number of photons required for direct absorption (truncated to an integer) with $\Delta E$ being the band-gap of material, $h$ is the Plank's constant, $\omega=2 \pi c / \lambda$ is the cyclic frequency of light of wavelength $\lambda, e$ is the electron charge, and $c$ is the speed of light. The electron quiver energy $\varepsilon_{o s c}=\frac{e^{2} E^{2}}{4 m \omega^{2}}$ with the electrical field strength defined by intensity/irradiance, $I_{p}$, as $E=\sqrt{I_{p} /\left(c \varepsilon_{0} n\right)}$, where $\varepsilon_{0}$ is the vacuum permittivity and $n$ is the refractive index of the host material. The electron-ion interaction is governed by the electron-phonon momentum exchange rate $v_{e-p h} \simeq 6 \times 10^{14} \mathrm{~s}^{-1}$ (Malinauskas et al., 2010); the exact value was not critically important for this qualitative estimation of the mechanisms discussed here.

With the multi-photon and avalanche ionization rates estimated by Eqs. (5) and (6) it is possible to calculate the rate of free electron generation, hence, the rate free radicals (broken chemical bonds) are created:

$$
\frac{d n_{e}}{d t}=n_{e} w_{i m p}+n_{a} w_{m p i}
$$

(a)

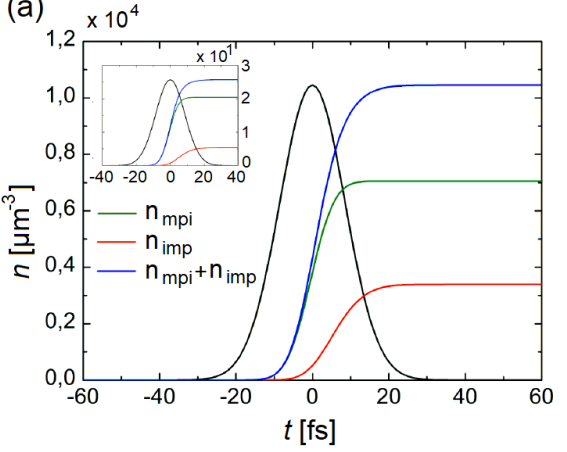

(b) $\times 10^{5}$

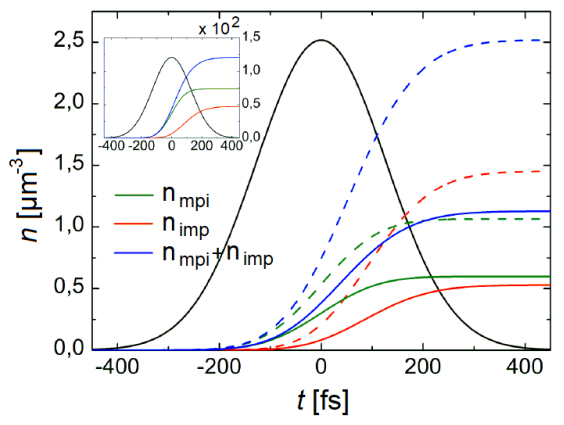

Fig. 3 Qualitative model of free electron generation dynamics due to the multi-photon and avalanche ionization $\left(n_{m p i}\right.$ and $\left.n_{i m p}\right)$ in PDMS pre-polymer. (a) Excitation: Ti:Sapphire $\left(\lambda_{1}=800 \mathrm{~nm}, \tau_{1}=20 \mathrm{fs}, I=5.2 \mathrm{TW} / \mathrm{cm}^{2}\right)$, photosensitized (and pure - inset) PDMS. b) Excitation: Yb:KGW $\left(\lambda_{2}=515 \mathrm{~nm}, \tau_{2}=300 \mathrm{fs}, I=1.2 \mathrm{TW} / \mathrm{cm}^{2}\right.$ - solid lines, $I=1.6 \mathrm{TW} / \mathrm{cm}^{2}$ dashed lines), photosensitized (and pure - inset) PDMS. Reproduced from (Rekštyte, 2016) with personal permission. 
where $n_{e}$ is free electron density (available only for the avalanche multiplication) and $n_{a}$ is the atom density (available only for the multi-photon ionization). Solution of the Eq. (7) is following:

$$
n_{e}(I, \lambda, t)=\left[n_{e 0}+\frac{n_{a} w_{m p i}}{w_{i m p}}\left[1-e^{-w_{i m p} t}\right]\right] e^{w_{i m p} t} .
$$

This equation allows to follow explicitly temporal evolution of ionization for the known temporal intensity envelope $I(t)$; here $n_{e 0}$ is the initial (dark) electron density in material $\sim 10^{10} \mathrm{~cm}^{-3}$. When second harmonic excitation is used, effects of long ns-background are suppressed which otherwise can significantly alter the avalanche ionization since the $w_{\text {imp }}$ scales as $\propto \lambda^{2}$ (Eq. 6).

For each individual material and exposure parameters, contribution of multiphoton and avalanche ionization as well as free electron density at the end of an incident laser pulse could be evaluated and compared. Example of such modeling, where free electron density dynamics in polydimethylsiloxane (PDMS) pre-polymer (both pure and photosensitzed) are evaluated as a result of excitation by laser pulses of different wavelengths and durations $\left(\lambda_{1}=800 \mathrm{~nm}, \tau_{1}=20 \mathrm{fs}\right.$ and $\lambda_{2}=515 \mathrm{~nm}$, $\tau_{2}=300 \mathrm{fs}$ ) and depicted in Fig. 3 (a) and (b) respectively. In both cases the dominant source of free electron generation is multi-photon absorption (green curves). However, the rate of avalanche ionization exceeds the rate of multi-photon absorptionby orders of magnitude $\left(w_{i m p}>>w_{m p i}\right)$ (see Table 1 for details). It is evident that the significance of contribution of avalanche ionization increases when the duration of exposing laser pulses is prolonged. At certain exposure conditions the free electron density grows large enough so that the avalanche ionization turns into the dominant uncontrolled process and may cause a breakdown of the material (Fig. 3 (b) dashed lines) (Rekštyte, 2016).

The same was found to be true for other polymer precursors popular in DLW nanolithography (i.e. SZ2080 and poly(ethylene glycol) diacrylate (PEG-DA)) (Table 1). In the narrowest band-gap material SZ2080 at the laser wavelength $\lambda_{0}=515 \mathrm{~nm}$, the ionization by a two-photon process $n_{p h}=2$ is expected. For the structures in SZ2080 obtained with $N A=1.4$ objective lens and $E_{p}=16.5 \mathrm{~nJ}$, the intensity $I_{p} \simeq 8.7 \times 10^{12} \mathrm{~W} / \mathrm{cm}^{2}$ (for 4-photon process at $1030 \mathrm{~nm}$ ). Correspondingly, the rates are: $w_{m p i} \simeq 3.4 \times 10^{10} \mathrm{~s}^{-1}$ and $w_{m p i} \simeq 8.4 \times 10^{13} \mathrm{~s}^{-1}$. At the $515 \mathrm{~nm}$ when two-photon absorption can be efficient and for $E_{p}=1.75 \mathrm{~nJ}$ the difference between the two ionization mechanisms becomes smaller: $w_{m p i} \simeq 3.7 \times 10^{10} \mathrm{~s}^{-1}$ and $w_{m p i} \simeq$ $4.4 \times 10^{12} \mathrm{~s}^{-1}$ (Buividas et al., 2013).

The contribution of avalanche ionization to the bond breaking and radicals' generation is essential for structuring pre-polymers in case of absence of dedicated photo-initiator molecules, or thermal-curing of resists, which normally require a post-exposure bake procedure. Those phenomena will be further discussed in later Sections 4 and 5. 
Table 1 Evaluation of multi-photon and avalanche ionization rates in various pre-polymers under different exposure parameters ${ }^{a}$.

\begin{tabular}{lllllll}
\hline$\lambda, \mathrm{nm}$ & Material & PI & $I_{p}, \mathrm{TW} / \mathrm{cm}^{2}$ & $n_{p h}$ & $w_{m p i} \times 10^{10} \mathrm{~s}^{-1} w_{i m p} \times 10^{10} \mathrm{~s}^{-1}$ \\
\hline 515 & PDMS & No & 1.6 & 3 & 0.004 & 299 \\
515 & PDMS & Yes & 1.2 & 2 & 2.65 & 374 \\
800 & PDMS & No & 5.2 & 4 & 0.019 & 2263 \\
800 & PDMS & Yes & 5.2 & 3 & 5.74 & 3772 \\
515 & SZ2080 & No & 1.7 & 2 & 3.70 & 440 \\
1030 & SZ2080 & No & 8.7 & 4 & 3.40 & 8400 \\
515 & PEG-DA-700 & No & 0.96 & 3 & 0.00075 & 170 \\
\hline
\end{tabular}

${ }^{a}$ Data collected from (Buividas et al., 2013), (Rekštyte et al., 2014a), (Rekštyte, 2016).

\section{Polymerization Without the Radical Photo-Initiators}

In multi-photon laser lithography process, a liquid or gel state monomers are converted into a solid state polymer under tightly focused light irradiation. The polymerization reaction begins with an initiation stage, during which certain materials, so called photo-initiators, decompose and form highly reactive species - radicals. Usually, chemical compounds with a low dissociation energy and high two-photon absorption cross-section are used to increase the efficiency of MPA polymerization. On the other hand, the vast majority of widely used commercial photo-initiators are adopted from the conventional lithography industry and were found to be cytotoxic (Raman and Bashir, 2015). This is one of the main drawbacks preventing possible applications of 3DLL formed micro-/nano-structures in biomedicine. Especially, taking into account biodegradable materials as they turn into initial compounds during the degradation process. On the other hand, once the photo-initiators are incorporated in 3D micro-objects they remain light absorbing species leading to

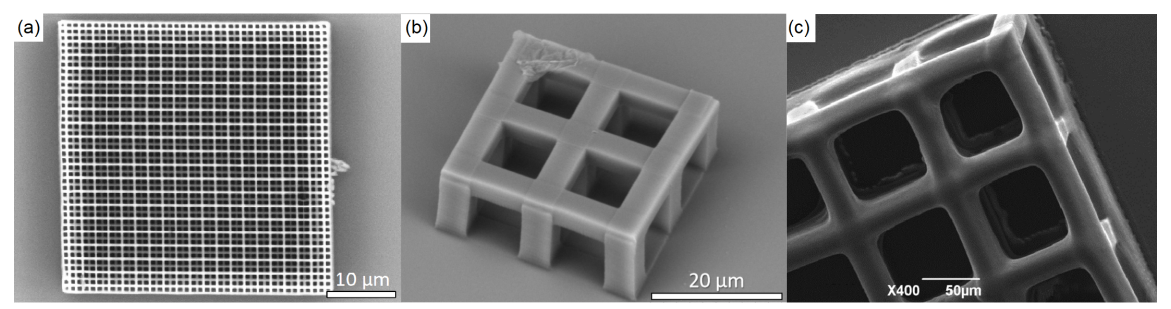

Fig. 4 3D functional structures produced in SZ2080, PDMS and GelMa pre-polymers without photo-initiators: (a) photonic crystal fabricated using $1 \mathrm{kHz}$ pulse repetition rate, $\lambda=1030 \mathrm{~nm}$ wavelength, $N A=1.4$ focusing and $16.5 \mathrm{~nJ}$ pulse energy. (b) Bioscaffold fabricated in PDMS using $\lambda=515 \mathrm{~nm}$ wavelength, $N A=1.25$ focusing, $2.5 \mathrm{~nJ}(0.5 \mathrm{~mW}), 2 \mathrm{~nJ}(0.4 \mathrm{~mW})$ and $1.5 \mathrm{~nJ}$ $(0.3 \mathrm{~mW})$ pulse energies for the upper layer,supporting pillars and the secondary scan, respectively and $0.1 \mathrm{~mm} / \mathrm{s} \mathrm{sample} \mathrm{translation} \mathrm{velocity.} \mathrm{Reproduced} \mathrm{from} \mathrm{(Buividas} \mathrm{et} \mathrm{al.,} \mathrm{2013)} \mathrm{with} \mathrm{permission.}$ (C)2013 Optical Society of America. (c) The 3D structures fabricated in GelMa, using $\lambda=520 \mathrm{~nm}$ wavelength, $N A=0.8$ focusing, $28 \mathrm{~nJ}$ pulse energy $(28 \mathrm{~mW})$ and $3 \mathrm{~mm} / \mathrm{s}$ writing speed. Reproduced from (Parkatzidis et al., 2018) with permission. (2)2018 WILEY-VCH Verlag GmbH \& Co. KGaA. 
induced losses detrimental for photonics and lowering the resilience to intense light which in turn limits the applicability in microoptics ${ }^{3}$. Finally, photo-initiators are not only efficiently absorbing but also highly fluorescing light, thus during the fluorescence microscopy the 3D object and the signal from region of interest are highly overlapping and potentially can not be distinguishable or spatially resolved. (Accardo et al., 2017).

\section{Structuring of the Pure Pre-Polymers}

Historically photo-initiators were believed to be an essential component for a nonlinear absorption and polymerization initiation. However, many studies have shown that, at certain exposure conditions, pure monomer materials without the presence of any photo-initiator can be reproducibly structured (Malinauskas et al., 2010; Buividas et al., 2013; Parkatzidis et al., 2018). In the earlier reports (i.e. (Takada et al., 2005)), structuring of pre-polymers with $0 \%$ photo-initiator was also mentioned, however, no further discussion on the underlying mechanism was provided.

In all cases, efficient structuring of non-photo-sensitized pre-polymers was achieved when relatively long $(\tau \sim$ sub-ps $)$ frequency-doubled pulses at moderate $\left(\sim 10^{5} \mathrm{~Hz}\right)$ repetition rate were employed for exposure. In contrast, applicability of ultra-fast oscillators, featuring pulse duration below $100 \mathrm{fs}, \lambda \approx 800 \mathrm{~nm}$ and repetition rate $\sim 80 \mathrm{MHz}$ found to be either very complicated (due to the shrinked processing window 4 ), or even impossible (Fischer et al., 2013; Rekštyte, 2016), depending on pre-polymer material. This tendency highlights the importance of

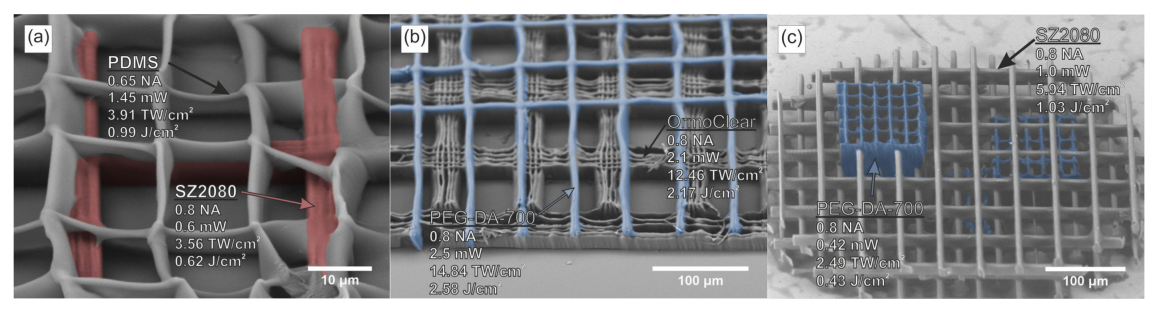

Fig. 5 (a) A composite scaffold fabricated out of hybrid organic-inorganic SZ2080 photopolymer (red) and polydimethylsiloxane (PDMS) without using photo-initiators. (b) A composite scaffold fabricated out of hybrid organic-inorganic OrmoClear and PEG-DA-700 photopolymers. (c) 3D composite scaffold fabricated out of SZ2080 and PEG-DA-700. PEG-DA-700 is colored blue in all pictures. Reproduced from (Rekštyte et al., 2014b) with permission. @2005 Japan Laser Processing Society.

\footnotetext{
${ }^{3}$ Resistance to intense light irradiation is commonly performed using Laser Induced Damage Threshold (LIDT) tests (Žukauskas et al., 2015)

${ }^{4}$ Processing window $\left(P W=I_{d} / I_{t h}\right)$ is the tuning range of light intensity between the polymerization threshold $\left(I_{t h}\right)$ and over-exposition (optical damage) $\left(I_{d}\right)$ causing micro-explosions in the material. Alternatively, a similar parameter denominated as Dynamic range $\left(D R=\left(I_{d}-I_{t h}\right) / I_{t h}\right)$ may be used in literature.
} 
controlled avalanche ionization mechanism, which is dominating as the light being localized in the dielectric material at a close to breakdown conditions. The possible explanation of this behavior relies on different ionization rates $w_{m p i}$ and $w_{i m p}$ as discussed in Sect. 3.

In case of pure monomer (e.g. PDMS) the linear absorption starts at $225 \mathrm{~nm}$ $(\Delta E=5.5 \mathrm{eV}$ ), thus, radiation of $\lambda=800 \mathrm{~nm}$ can be absorbed only via 4-photon process, which is, indeed, low-probability event. The free carrier's generation rate due to multiphoton-absorption is $\sim 5$ orders of magnitude lower compared with avalanche ionization rate. Thus, as a very few free electrons are generated within irradiated volume $\left(\sim 10^{1}\right)$ and a pulse durationis too short for avalanche to proceed. As a result, a final electron density is too low to initiate polymerization reaction (see Fig. 3 (a) inset). On the contrary, the situation for a $515 \mathrm{~nm}$ amplified sub-ps pulse is different. The radiation can be absorbed via 3-photon process, and the avalanche ionization rate is lower by order of magnitude compared to the NIR illumination. In this case, the free radicals density increases up to $\sim 10^{2}$ per pulse, leading to photo-polymerization reaction (see Fig. 3 (b) inset). Moreover, it was demonstrated, that an absence of any photo-initiators leads to increased structuring resolution in pure organic-inorganic SZ2080 resist (Malinauskas et al., 2010).

Until to date, 3D structuring without photo-initiators was demonstrated in different classes of materials, namely hybrid sol-gels, organosilicons (PDMS), hydrogels (PEG-DA, gelatin methacrylamide (GelMa)) (Fig. 4) or even compositions of them (Fig. 5). 3D micro-structured polymer scaffolds for tissue regeneration were fabricated and tested both in vitro (Selimis et al., 2015) and in vivo (Mačiulaitis et al., 2015).

Besides the manufacturing of bio-compatible scaffolds, other application areas may benefit from the absence of photo-initiators as well. Since the DLW lithography is used for production of micro-optical components, optical properties of photocurable materials is of high importance. For instance, the elimination of photoinitiators is a straightforward way to improve optical quality of polymers as the transmittance range increases (Fig. 6). Moreover, non-photo-sensitized polymers demonstrates higher Laser Induced Damage Threshold (LIDT). Indeed, up to $38 \%$ higher LIDT was measured for the pure SZ2080 polymer, compared to photoinitiated $\left(14.72 \mathrm{~J} / \mathrm{cm}^{2}\right.$ and $10.63 \mathrm{~J} / \mathrm{cm}^{2}$ correspondingly), under ns pulses exposition at $1030 \mathrm{~nm}$. Even more remarkable difference by an order of magnitude in LIDT values was observed when materials exposed by $532 \mathrm{~nm}$ pulses $\left(\left(13.67 \mathrm{~J} / \mathrm{cm}^{2}\right.\right.$ versus $1.43 \mathrm{~J} / \mathrm{cm}^{2}$ ) (Žukauskas et al., 2015). This is in line with for qualitative evaluation conducted by Jonušauskas et al. (2017), where LIDTs of $\mu$-lenses were investigated.

\section{Structuring of Functional Additives Doped Resins}

The trending research direction in the field of DLW lithography is further pursuance of new photo-curable composite materials exhibiting unique physical features. Functional doping of pre-polymers prior to DLW lithography was demonstrated for wide spectra of applications. For instance, mixing pre-polymers with metal oxide NPs 
increases refractive index of final structures (Duan et al., 2004; Guo et al., 2014), introduction of up-converting luminescence NPs enables manufacturing of thermosensitive micro-patterns (Wickberg et al., 2015), while dispersed carbon nano-rods leads to embedded nano-wires, self aligned inside polymer structure (Ushiba et al., 2013). In recent papers, additives like quantum dots (Peng et al., 2018) or metal NPs (Jonušauskas et al., 2016) were found either to alter structuring resolution or polymerization threshold. Indeed, the possibility to enhance pre-polymer photosensitivity (i.e. by presence of non-toxic Au NPs) is a promising pathway towards efficient manufacturing of bio-compatible/bio-degradable structures.

\section{The Temperature Effects on Nano-Polymerization Process and Polymerized Structures}

The thermal effects indeed act an important role refashioning the mechanism of nano-polymerization. It is intuitively clear, that the radical diffusion and chain termination reactions, taking place during polymerization, are temperature dependent. Both ambient thermal conditions and local temperature gradients, induced by the writing process itself, are expected to impact the pathway of polymerization and consequently, affect the morphology of structures fabricated. The quantitative investigation of local temperature changes within microscopic volumes of polymer is complex to carry out both theoretically and experimentally. Heat transfer kinetics inside nano-patterned polymer sample is proved to be different in comparison with a bulk medium (Morikawa et al., 2009). Moreover, the dominant heat sources during the nano-polymerization reaction remains under debates, as evidently, they are material and excitation parameters dependent (as discussed in Sect. 3). No conventional temperature acquisition approaches can be applied for direct measurements as the thermal gradients on a micrometer scale must be resolved. Non-contact optical temperature measurement methods (i.e. luminescence spectra acquisition from dispersed up-converting nanoparticles (UCNPs)) is believed to be a promising manner as was successfully applied previously for sensitive temperature imaging (i.e. in live cells (Vetrone et al., 2010)). However, engineering challenges arises during the real-life experiment as a superior co-alignment of the writing and probing beams is essential to avoid spatial averaging of a measured signal (see further discussion for

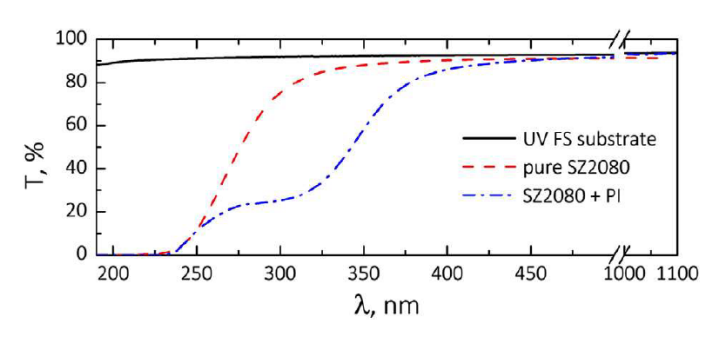

Fig. 6 The transmittance spectrum of the photosensitized and non-photosentisized SZ2080 thin films (black solid curve shows the transmittance of the UV Fused Silica substrate). Reproduced from (Žukauskas et al., 2015) with permission. (C)20014 Elsevier B.V. 
details). Also, the special care should be taken, so that the probing radiation would not alter process conditions at a given writing parameters and vice versa.

All those above-mentioned obstacles likely is the reason, why the very few studies of temperature-related properties of DLW polymerization are present in a scientific literature. Also, one should note, that individual research groups conduct experiments using different DLW setups and pre-polymer materials, thus, straightforward juxtaposition of the results is not always possible. In this section we try to collect those small pieces of a wide picture, having the aim to emphasize the diversity and contradictions of experimental data rather than propose the comprehensive answers and conclusions. On the other hand, this is inviting for further, more systematic studies of temperature-related effects.

\section{Local Temperature Changes During the DLW 3D Nano-Polymerization}

To the best of our knowledge, the very first experimental effort to measure absolute values of temperature during DLW polymerization was reported in (Mueller et al., 2013). Pentaerythritol triacrylate (PETA) monomers photosensitized by Irgacure 819 photo-initiator and co-doped with $\mathrm{NaYF}_{4}: \mathrm{Yb}^{3+}(20 \%)$; $\mathrm{Er}^{3+}(2 \%) \mathrm{UC}-$ NPs as thermo-sensitive agents was exposed by Ti:Sapphire oscillator $(810 \mathrm{~nm}$, $80 \mathrm{MHz}, 100 \mathrm{fs}$ ). Meanwhile, the sample was probed co-axially by CW laser beam $(980 \mathrm{~nm})$ for UCNPs excitation and the luminescence spectra were obtained as they are temperature dependent (see the Ref. for more experimental details). Authors report $<5 \mathrm{~K}$ temperature increment, while exposition parameters remains within processing window, and significant temperature increase (by $\Delta T \approx 300 \mathrm{~K}$ ) was observed as soon as micro-explosions start to occur (Fig. 7 (a)). The results were found to be consistent with numerical computations performed, as heating by $5-10 \mathrm{~K}$ was
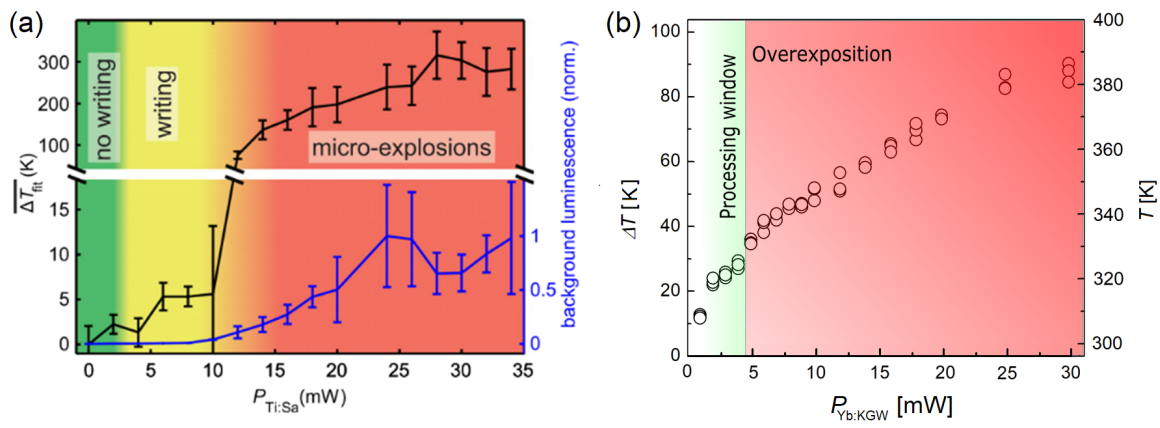

Fig. 7 (a) Fitted average temperature as a function of the writing laser power $P_{T i: S a}\left(P_{980 \mathrm{~nm}}=\right.$ $17 \mathrm{~mW}$ ) for the three different regimes. The integration time was 20 s except for temperature measurements with $P_{T i: S a}=10 \mathrm{~mW}$, where it was increased to $400 \mathrm{~s}$ for better accuracy. Reproduced from (Mueller et al., 2013) with permission. (C2013, AIP Publishing LLC. (b) Fitted average temperature as a function of the laser exposure intensity for different fabrication regimes from below threshold to over-exposition (unpublished data from authors). 
expected due to excess energy of polymerization reaction. Surprisingly, the same tendency was observed independently on pulse repetition rate, which was varied in a wide range ( $1 \mathrm{kHz}-80 \mathrm{MHz})$ (Mueller et al., 2016).

Later, the experiment was replicated by the authors of this Chapter, adopting very similar approach for luminescence spectra acquisition from the micro-volume surrounding a polymerization reaction zone. Amplified Yb:KGW laser $(1030 \mathrm{~nm}$, $200 \mathrm{kHz}, 300 \mathrm{fs}$ ) was employed for writing and several types of UCNPs with different host matrix and size, tested as optical nano-thermometers, dispersed in pure hybrid SZ2080pre-polymer (see (Varapnickas et al., 2018) for details). In a slight contrast with Mueller and coworkers' study, the gradual temperature increase (up to $\Delta T \approx$ $30 \mathrm{~K}$ ) was observed within normal writing conditions, and only marginal step-like increment detected when over-passing the optical damage boundary. The stronger heating could be attributed to the increased contribution of controlled avalanche ionization, as the laser pulse duration is prolonged. However, we admit the fact that the measured values are averaged in time (spectra integration duration) and space (possible mismatch between writing and probing volumes) thus, can be significantly lower than actual peak temperature values.

The role of thermal accumulation also has been evaluated indirectly, i.e. as a feature size dependence on laser pulse repetition rate. Baldacchini and co-workers have performed an experiment in which suspended lines have been written maintaining the same net fluence but varied time-gap between arrival of fs pulse bursts. The sequences of constant duration $(1 \mu \mathrm{s})$ and variable repetition rate bursts were picked out of a pulse-train from Ti:Sapphire oscillator $(800 \mathrm{~nm}, 80 \mathrm{MHz}, 100 \mathrm{fs})$ focused to the pre-polymer via DLW system. A sharp increase in line-width (decrease of structuring resolution) was observed at $0.05 \mathrm{MHz}-0.10 \mathrm{MHz}$ burst repetition rate (corresponding to 19 - $9 \mu$ s duration between subsequent bursts). This phenomenon was attributed to decreased contribution of thermal polymerization as a cooling time of exposed volume predicted to be $\sim 16 \mu$ s (Baldacchini et al., 2012).

The effects of thermal accumulation on MPA nano-polymerization have been investigated also by using a diode-pumped picosecond Nd:YVO laser with cavity dumping. Specifically, the fundamental $(1064 \mathrm{~nm})$ and the frequency-doubled $(532 \mathrm{~nm})$ wavelengths were used for fabrication using laser pulses ranging from 8 to $25 \mathrm{ps}$ and repetition rates from 0.2 to $1 \mathrm{MHz}$ (Malinauskas et al., 2011). In a good agreement with Baldacchini's study, thermal accumulation became significant and influenced structuring resolution only when the cooling time of the irradiated spot became comparable to the time separation between consequent pulses.

Moreover, Seet et al. (2006) observed thermal annealing process when exposing epoxy based photoresist SU-8 by 180 fs pulses. A conceptually new approach of thermally enhanced polymerization, which is different from the common view of a non-linear two-photon absorption dominance in photo-polymerization by femtosecond pulses, was introduced. Authors justified the polymerization process by the possibility of cumulative direct absorption of black-body emission from hot electrons, generated via avalanche ionization. It was calculated that a $0.5 \mathrm{~nJ}$ pulse generates an equilibrium temperature of $227^{\circ} \mathrm{C}$ within the focal volume (assuming insufficient time for heat diffusion). 
Lastly, the similar thermo-/photo-curing effect in SU-8 resin was observed by Nguyen et al. (2016). The sample was exposed by CW laser operating at $532 \mathrm{~nm}$ (several $\mathrm{mW}$ of output power) while scanning at very low translation velocity $(1 \mu \mathrm{m} / \mathrm{s})$. This is required for thermal accumulation at low optical linear absorption. Surprisingly, relatively high resolution structures (comparable to those obtained by MPA 3D nanopolymerization) were produced in that work.

The Effect of Ambient Temperature on Photo-polymerization

No less important and interesting are the effects on DLW polymerization caused by the varied ambient (global) temperature of the pre-polymer. Back in 2008, Takada and co-workers showed that during exposition of the frozen SCR500resin $(T<$ $-50^{\circ} \mathrm{C}$ ) radical generation is not prevented, but the polymerization is slow until the temperature is increased to $>10^{\circ} \mathrm{C}$ (Takada et al., 2008). On the other hand, it is known that the thermal polymerization takes place if the ambient temperature of the monomers increases (typically around $140^{\circ} \mathrm{C}$ ) (Ong et al., 2006; Suzuki et al., 2012). The question of interest is if the variation of ambient temperature in between those extremes may contribute to improved spatial resolution of the structures fabricated.

The subject was addressed in the study by Takada et al. (2008), where the entire sample of SCR 500 resin was thermally regulated from -50 to $80^{\circ} \mathrm{C}$ and minimal feature sizes of structured lines were analyzed. It has been expected that decreasing the temperature of photo-polymerization may notably improve the spatial resolution due to reduced radicals' diffusion and Brownian motion of small molecules. However, only a marginal improvement of writing resolution by the rate $\sim 0.8-1.1 \mathrm{~nm} /{ }^{\circ} \mathrm{C}$ was observed while decreasing the temperature from $20^{\circ} \mathrm{C}$ to $-50^{\circ} \mathrm{C}$. On the contrary, distinct increase in writing resolution (i.e. reduction of line-width by $\sim 27 \%$ ) was achieved while heating the sample up to $80^{\circ} \mathrm{C}$. Such behavior was justified by the well-known effect of temperature accelerated termination of the polymerization reaction. Evidently, these findings are consistent with Mueller and co-workers' observation, that the writing threshold power increases by $1.4 \% \mathrm{~K}^{-1}$ as temperature of PETA pre-polymer increases (from $10^{\circ} \mathrm{C}$ to $80^{\circ} \mathrm{C}$ ) (Mueller et al., 2013).

After all, the only reasonable conclusion can be drawn is that the results, obtained from experiments up to date, can not be generalized by a single, well described model of temperature kinetics. Likely, the particular behavior is both material and light parameters dependent. Thus, further investigation of temperature effects in different pre-polymers and under different exposure conditions remains of high importance.

\section{Light Polarization Induced Effects on 3D Nano-Polymerization Process}

A systematic study of polarization control in light assisted additive manufacturing of polymers is still limited and importance of light polarization is barely mentioned in 
the scientific articles in the field of DLW in pre-polymers. This could be explained by the fact that polymers shrink (especially in nano-scale), wrinkle and tend to show high plasticity, thus, loosing their DLW defined shape and feature rigidness. As a result, structural changes induced by light polarization variation are not easy to indicate, unless special attention is being payed. Sun et al. (2003) previously showed that using linearly polarized beam leads to the formation of voxels which are elongated in the direction of the electric field vector, while they are symmetric in both $X$ and $Y$ directions when circular polarization is used. However, no in-depth investigation of this effect on the fabrication of functional structures was carried out in that work. On the other hand, the influence of laser radiation's polarization to the subtractive femtosecond processing of various materials has been widely studied. It can manifest in the formation of nano-ripples, which are mostly perpendicularly oriented in respect to the electric field vector, when surface ablation of metals, semiconductors, dielectrics or polymers is being performed. The ablation threshold and the pattern of ablated holes are also influenced by the electric field vector direction of the laser beam (Venkatakrishnan et al., 2002). The control of light wave vibrations direction is essential for defining local form birefringence and patterning of optical anisotropy while structuring glass by nano-gratings (Beresna and Kazansky, 2010). The experimental validation and in-depth discussion about polarization influence on feature size of fabricated structure in hybrid organic-inorganic pre-polymer SZ2080 can be found elsewhere (Rekštyte et al., 2016). In this Section we broaden the outlook by presenting the results acquired by changing photo-initiation conditions in SZ2080 as well as investigating other popular pre-polymers (OrmoComp, PETA, PEG-DA) widely used in DLW nano-lithography experiments.

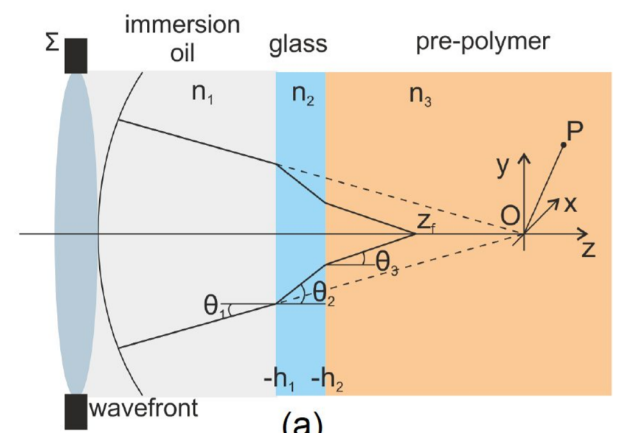

(a)

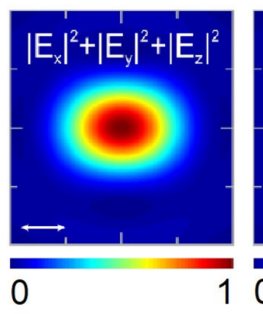

(b)

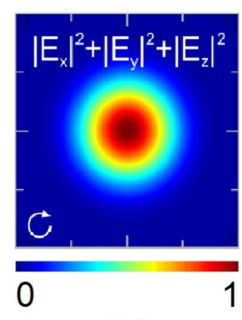

(c)

Fig. 8 (a) Focusing of the laser beam through a three-layer media. (b)-(c) Results of the theoretical modeling of the intensity distribution (SZ2080, $\lambda=1030 \mathrm{~nm}, N A=1.4$ ): the sum electric field when the focused beam is linearly (horizontally) polarized (b) and when it is circularly polarized (c). Adapted from (Rekštyte, 2016) with personal permission. 
Theoretical Model

The dependence of spatial resolution of the fabricated elements on the laser beam polarization can be explained by introducing the theory of focusing, especially if objectives with high $N A$ are used and the paraxial approximation is no longer valid (Sun et al., 2003). The model based on a vectorial Debye theory was adopted to calculate the point spread function (PSF) of light which is being focused through a stratified medium, i.e. immersion oil, glass substrate and pre-polymer (Fig. 8(a)). An optical system having an optical axis $z$ is considered. In such environment the light crosses two interfaces - immersion oil/glass in the $z=-h_{1}$ plane and glass/prepolymer in the $z=-h_{2}$. It is assumed that the layers are homogeneous and isotropic, and their refractive indices are $n_{1}$ (immersion oil), $n_{2}$ (glass) and $n_{3}$ (pre-polymer) respectively. The linearly polarized (along $x$ direction) monochromatic and coherent electromagnetic wave is emitted from a point source at $z=-\infty$. It falls onto the aperture $\Sigma$ (lens) which creates a converging wave front in the image space. The origin of the coordinate system coincides with a corrected Gaussian focal point (the point in which the radiation would focus if all conditions would meet the design parameters of objective). The electric field is determined at the point $P(x, y, z)$ which is close to the focal point. It is assumed that the size of the aperture and its distance from the point $P$ are large compared to the wavelength. The results of intensity distribution calculations for SZ2080 pre-polymer when the laser beam $(\lambda=1030 \mathrm{~nm})$ is focused by a $N A=1.4$ objective are presented in Fig. 8 (b)-(c). An elongation in the direction of the electric field vector for linearly polarized light is observed (Fig. 8(b)) while a symmetrical intensity distribution is obtained when a circularly polarized beam is being focused (Fig. 8(c)). Asymmetrical distribution can be explained by the pronounced $E_{y}$ and $E_{z}$ components, the latter of which has the biggest influence to the loss of symmetry in the intensity distribution since its maximum value reaches up to $16 \%$ of the $\left|E_{x}\right|$ component.

The calculated ratios of intensity distribution profiles for different pre-polymers (FWHM values in $X$ and $Y$ axes) are given in Table 2. Some discrepancy between calculated and experimentally measured line-width ratios is expected as only a single point exposure is considered in the model, while an overlap of pulse trains occurs during laser writing. For correct interpretation of the line formation dynamics an integrated intensity distribution along the sample translation direction should be considered. If the sample is translated along the electric field vector, an integrated intensity profile has higher peak power but is narrower than in the perpendicular direction. Analysis of these profiles shows that there is a point of intersection, meaning there is an intensity value, at which the line would be formed of the same width regardless of translation direction. Also, the ratio of line-widths should depend on the radiation intensity. 
Table 2 Theoretical calculation results for various pre-polymers. Numerical aperture of objective lens is constant $(N A=1.4)$.

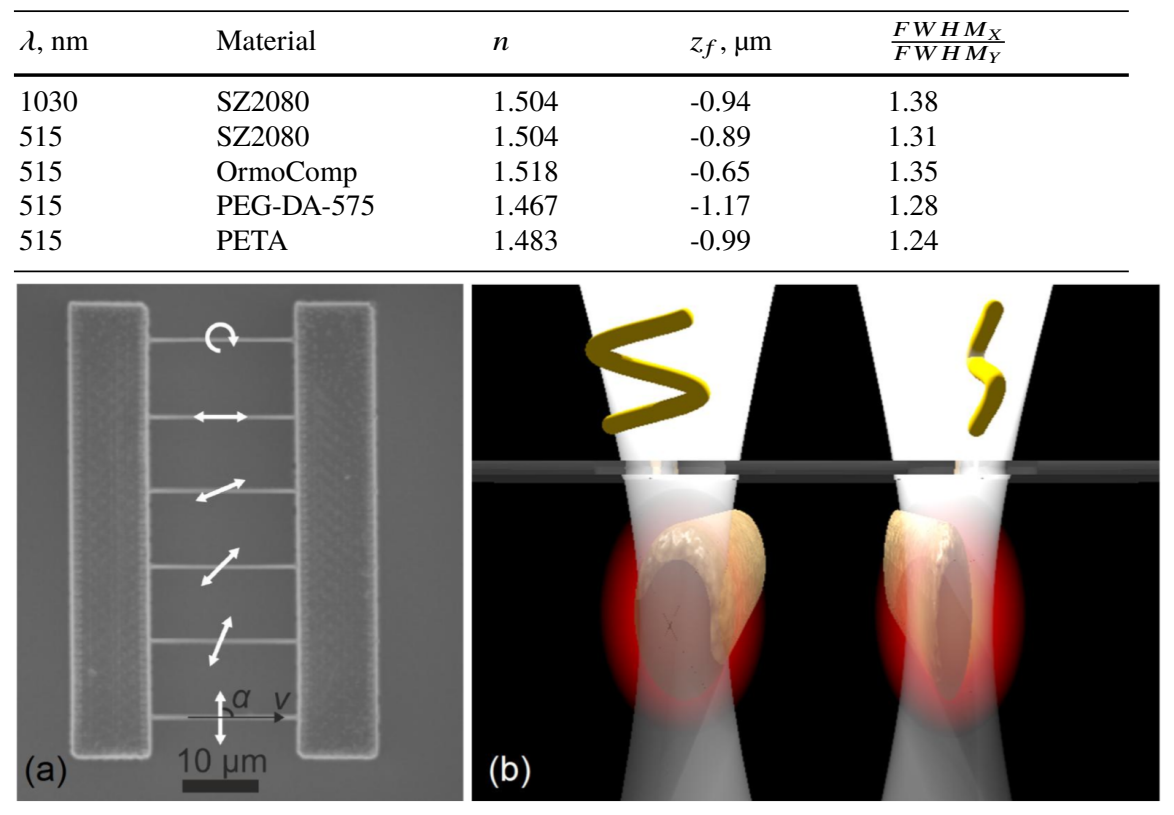

Fig. 9 (a) SEM micrograph of suspended lines structure used for evaluation of lateral structuring resolution. White arrows indicate the polarization direction. Adapted from (Rekštyte, 2016) with personal permission. (b) Visualization of different angles of polarization vector in respect to scanning direction: $\alpha=90^{\circ}$ - left, $\alpha=0^{\circ}$ - right.

\section{Experimental Observations}

The investigation was conducted using Yb:KGW laser based DLW 3D nanolithography system $(\tau=300 \mathrm{fs}, v=200 \mathrm{kHz}, N A=1.4)$. Structures, consisting of lines, suspended between two rigid walls (see Fig. 9(a)) written while varying the angle $\alpha$ between the polarization vector and sample translation direction $(v=100 \mu \mathrm{m} / \mathrm{s}$ ), were fabricated. The pre-polymers studied were: SZ2080 (pure and doped with 1 wt.\% Irgacure 369 (IRG)), OrmoComp, PEG-DA-575 + 1 wt.\%, IRG, PETA $+1 \mathrm{wt} . \% \mathrm{IRG}$ for structuring at $\lambda=515 \mathrm{~nm}$ wavelength and SZ2080 $+1 \mathrm{wt} . \%$ 4,4'-bis(diethylamino)-benzophenone (BIS) for $\lambda=1030 \mathrm{~nm}$. It was empirically confirmed that the suspended lines fabricated out of SZ2080 (Fig. 9 (a)), OrmoComp and PETA pre-polymers (not shown) are thickest when the angle between the electric field vector and the sample translation direction is $\alpha=90^{\circ}$ (Fig. 9 (b) left) and thinnest when $\alpha=0^{\circ}$ (Fig. 9 (b) right). Otherwise, the axial resolution does not depend on the angle $\alpha$.

The obtained experimental results were consistent with theoretical simulation: feature size variation versus polarization vector angle $\alpha$ was material dependent and measured to be from 17 to $22 \%$ of the line-width for SZ2080 resins (pure and 
photo-sensitized) (Fig. 10). The similar line-width variation, although noticeably smaller in magnitude, was observed when processing OrmoComp (Fig. 11 (a)) and PETA (Fig. 11 (b)) pre-polymers (5\% and $7 \%$ variation respectively). In contrast, no clear line-width dependence on the angle $\alpha$ was observed for the PEG-DA-575 pre-polymer (Fig. 11 (c)). This might be explained by highly concealed dependence due to the high shrinkage of soft hydrogel material. However, this polymer precursor exhibited a different response to the polarization variation, i.e. a shrinkage of the fabrication window (or rather the decrease of damage threshold value). Furthermore, lines fabricated using circular polarization were the thinnest for all pre-polymers investigated as well as the highest axial resolution was achieved. Such behavior can be attributed to the possibly different multi-photon absorption cross-sections for linearly and circularly polarized light.

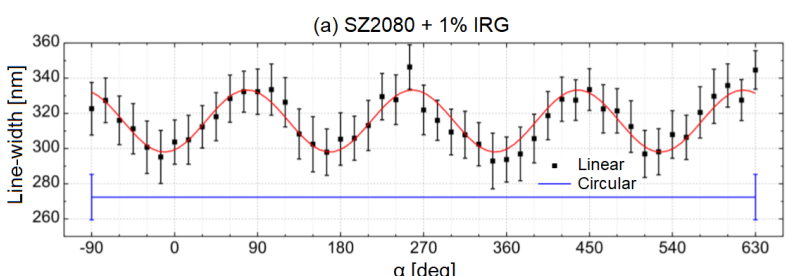

Fig. 10 The dependence of the suspended lines lateral dimensions on the angle between the electric field vector of linearly polarized beam
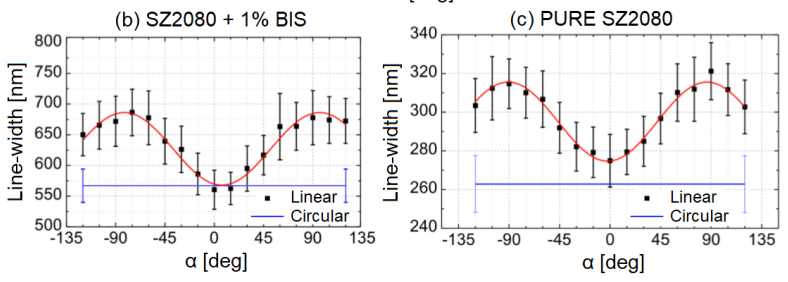
and the sample translation direction (approximated by a $\sin (x)$ function) in (a) SZ2080 + IRG; (b) SZ2080 + BIS; (c) pure SZ2080 pre-polymers. The error bars show the standard deviation. Adapted from (Rekštyte, 2016) with personal permission.
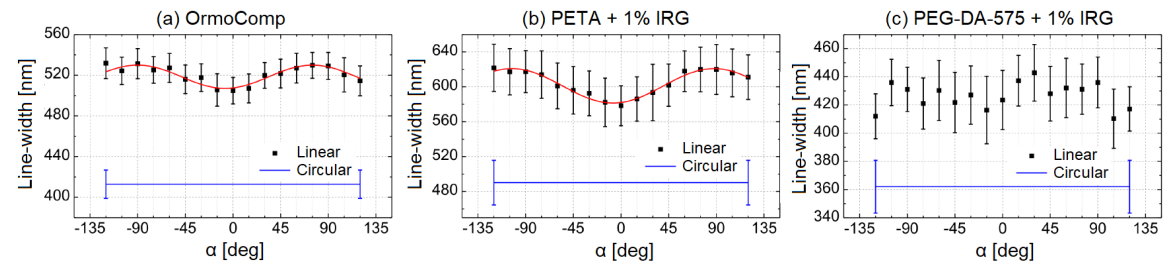

Fig. 11 The dependence of the suspended lines lateral dimensions on the angle between the electric field vector of linearly polarized beam and the sample translation direction (approximated by a $\sin (x)$ function) in (a) OrmoComp; (b) PETA; (c) PEG-DA pre-polymers. The error bars show the standard deviation. Adapted from (Rekštyte, 2016) with personal permission.

It could be concluded, that the impact of polarization direction depends on chemical (molecular structures) and physical (shrinkage and mechanical rigidity) material properties. Polarization influence is more significant for harder polymers (i.e. hybrid 


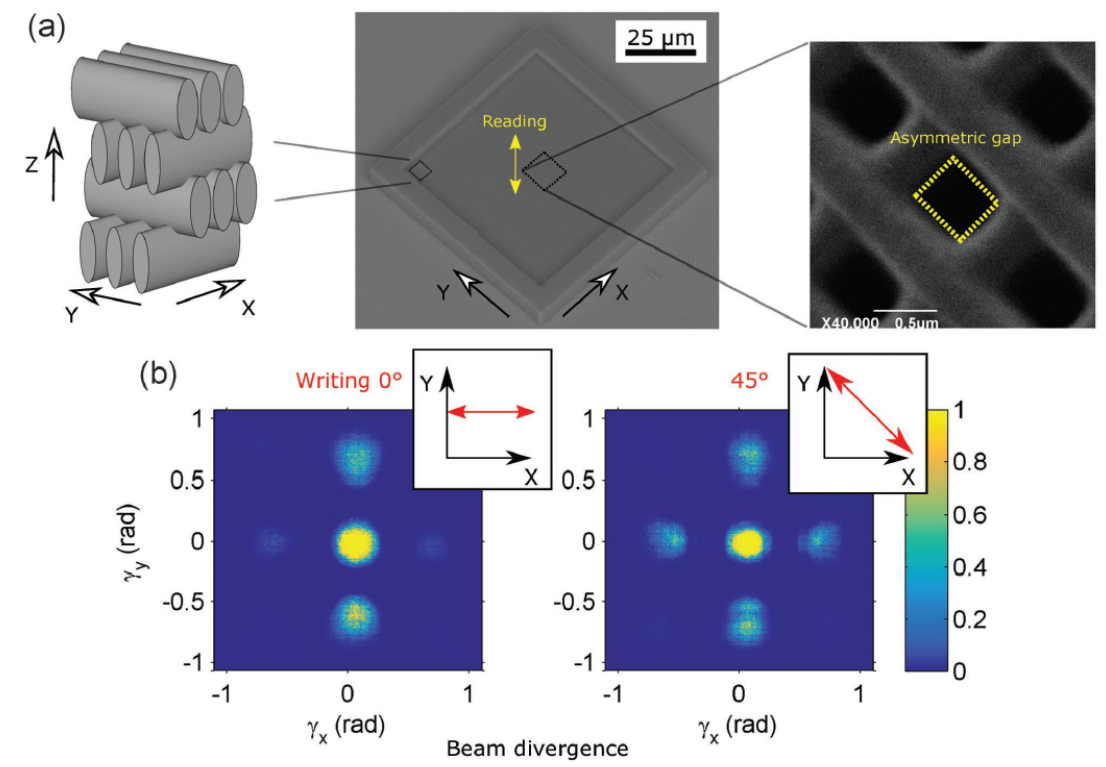

Fig. 12 (a) SEM micrograph of a woodpile photonic crystal (PhC). The line-width difference due to polarization is $8 \%$ resulting in an asymmetric gap shown at top right. (b) Schematic shows the diffraction pattern obtained by focusing a Gaussian He-Ne probe beam $(\lambda=633 \mathrm{~nm})$ on the $\mathrm{PhC}$ and projecting the resulting pattern onto a screen. An asymmetrical diffraction pattern for the DLW polarization oriented along the $\mathrm{PhC} X$-axis is clearly recognizable on the left side, whereas it is symmetrical if pre-compensated by writing direction on the right side. Reproduced from (Rekštyte et al., 2016) with permission. (C2016 WILEY-VCH Verlag GmbH \& Co. KGaA, Weinheim.

organic-inorganic) compared to the soft polymers (pure acrylates, hydrogels). As only the lateral resolution of the fabricated lines is affected by the direction of linear polarization vector, polarization control may be used for dynamic tuning of the aspect ratio of the structured elements. Indeed, the slight line-width variation is not crucial as long as components, constructed of relatively thick building blocks (i.e. scaffolds, volumetric optical components, etc.), are being fabricated. However, one should pay a special attention to polarization dependent line-with in case fine structures consisting of high resolution elements (i.e. phase gratings, photonic crystals) $(\mathrm{PhC})$ are considered. As an illustration, there is noticeable difference in performance of $\mathrm{PhC}$ (Fig. 12) if no polarization control or geometry pre-compensation is performed.

\section{Diffusion Assisted Radical Quenching}

As it was over-viewed in the previous sections, the resolution of DLW lithography might be adjusted by tailoring parameters of the writing beam (i.e. wavelength, 
pulse repetition rate, pulse duration, exposition dose, polarization, etc.). However, it is not easy to achieve less than $100 \mathrm{~nm}$ structuring resolution via control of the laser parameters only. The previous studies revealed that the structuring resolution can be further increased by intentionally introducing radical quenchers in the prepolymer (Takada et al., 2005; Park et al., 2006; Sakellari et al., 2012). Later on the comprehensive theoretical study of diffusion assisted radical quenching method, focusing on the resolution capabilities and optimal writing parameters was conducted by Pikulin et al. (2015).

The principle of diffusion assisted radicals quenching mechanism is illustrated in Fig. 13. The density of radicals generated within exposed volume is proportional to the square of the photon flux $(N)$ (see eq. 2), whereas the intensity is the function of radius to the axis of the laser beam and the propagation distance among the axis (eq. 1). Therefore, produced radicals are situated within a volume, defined by contour of ellipsoid (Fig. 13 inset A), with the highest density in the very central area and continuously decreasing in the surroundings. In case of presence of the quenching species, inhibiting polymer chains from growing long, the degree of cross-linking in an outer regions becomes insufficient and only the central part of exposed volume sustains the wet development process (Fig. 13 inset B). As a result, the feature size of voxel is reduced.

In 2005, Takada and co-workers demonstrated the reduced minimum feature size of the polymerized lines from $120 \mathrm{~nm}$ to around $100 \mathrm{~nm}$ by introducing as low as $0.8 \mathrm{wt} . \%$ of radical quencher into SCR500 resin. Authors pointed out, that $100 \mathrm{~nm}$ is not the lowest limit of structuring resolution, however further reduction of linewidths would lead to softer polymerized parts and less clear interface between the liquid and solid phases (Takada et al., 2005). A year later, Park et al. (2006) published a study on the quenching effect when different concentration of radical quencher 2,6di-tert-butyl-4-methylphenol (DBMP) present in SCR500 pre-polymer. The lines of slightly below $100 \mathrm{~nm}$ in width were fabricated out of the resin containing $5 \mathrm{wt} . \%$ of quencher. In addition, a quantitative evaluation of reduced elastic modulus (by $\sim 31 \%$ ) and hardness (by 34\%) was presented. However, no increased-resolution $3 \mathrm{D}$ structures or reduced minimal distance between high-resolution lines (which is

Fig. 13 Schematic illustration of radical quenching mechanism. In case the radical quencher is mixed into the original resin, the voxel size $\left(C_{b}\right)$ is reduced comparing to the case of the original resin only used $\left(C_{a}\right)$. Adapted from (Park et al., 2006) with permission. (CThe Polymer Society of Korea and Springer 2006

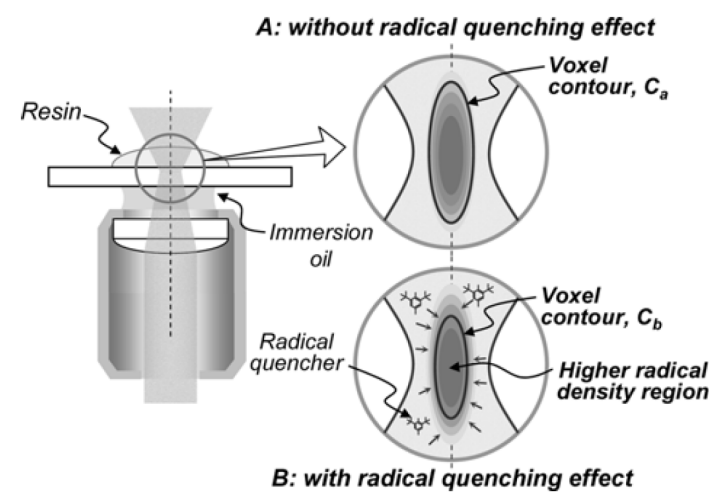


set by so called "memory effect" (Fischer and Wegener, 2011) was demonstrated in that work. Both works suggest, that the reduction of minimal feature size is possible at an expense of reduced polymerization degree. As the radical quenchers prevent a chain grow reaction, the structures fabricated consists of shorter polymer chains (smaller molecular weight) which results in tendency to higher shrinkage of the structure during the development phase. Also, the reduced mechanical strength of fabricated structures was acknowledged in both works. However, a possible overcome by introduction of post-print UV-curing step (Oakdale et al., 2016) was proposed. Unreacted acrylate groups could be polymerized during this procedure, thereby furthering the degree of conversion and increasing cross-link density.

In more recent work, Sakellari et al. (2012) demonstrated fabrication of 3D PhC woodpile structures with $400 \mathrm{~nm}$ intra-layer period out of hybrid organic-inorganic material SZ2080. Amine-based monomer 2-(dimethylamino) ethyl methacrylate was mixed into pre-polymer as a radical quencher. In comparison, using a similar material without any inhibitor, the lowest intra-layer period for woodpile reported was $900 \mathrm{~nm}$. Authors also claimed that the presence of this quencher increased the writing resolution without compromising the mechanical stability of the structures. Although, no experimental evaluation of the mechanical strength was provided in the paper.

The main achievement demonstrated in this work was the reduction of the "memory effect". Due to the relatively low scanning speed, the diffusion of the quenching molecules was capable in time period between the writing of two adjacent lines. Indeed, during the first irradiation by the Gaussian beam, the concentration of a quencher diminishes to zero at the center of the focal area and only fractionally alters at the periphery. Given that a slow scanning speed is employed in the writing process, the quenching molecules diffuse through the whole focal area, recovering its concentration homogeneous everywhere. When the subsequent line is being structured, radicals neither from the photo-initiator nor from the polymer remain since they were inhibited by the quenching molecule. The polymerization processes for each fabricated line thus, can be considered independent from any other, minimizing the "memory effect" and allowing fabricating structures closer to each other.

The effect of diffusion assisted quenching is prominent as long as slow scanning speeds are used (e.g. $20 \mu \mathrm{m} / \mathrm{s}$ in a particular work) allowing enough time for quencher to diffuse. In case of exploitation a modern DLW setup, empowering fabrication at much higher scanning speeds (i.e. $\sim \mathrm{mm} / \mathrm{s}$ ), diffusion assisted quenching approach becomes inapplicable. Even so, authors propose a work-around by employing a writing strategy of multiple scans. There, each layer of the structure could be scanned multiple times at a fluence below the polymerization threshold. Some monomer/oligomer units would be polymerized during each scan, however not enough to form a solid structure. During the time it takes to scan the whole layer, the quencher would diffuse. Eventually, after multiple scans, there would be enough polymerized material for the structure to survive after development (Sakellari et al., 2012). 


\section{Future Challenges and Perspectives}

The obtainable resolution in DLW 3D nano-lithography is mainly determined by the spatio-temporal localization of photons and their interaction with matter. During the two decades span of technology's evolution, a significant progress was made in both directions pushing the resolution limits well below $100 \mathrm{~nm}$ benchmark. For example, stimulated emission depletion (STED) technique was transferred from optical nanoscopy to DLW materials processing, enabling further reduction of structuring resolution (Fischer and Wegener, 2011). Indeed, some technological advances, proved to be effective in laboratory conditions, have very limited potential for industrial applicability due to their complexity and stability and (or) repeatability issues.

A significant advance in photo-sensitization of pre-polymer materials are being made as efficient water soluble and non-toxic photo-initiators are being developed ( $\mathrm{Li}$ et al., 2013) In parallel the approach to 3D nanostructure using inorganic dopants, such as Au-nanoparticles (Jonušauskas et al., 2016), or even photo-structuring of pure (Malinauskas et al., 2010) materials is widening its implementation employing fs-pulsed lasers. It enables precise 3D fabrication of functional materials targetted for applications without the need to special preparation making them photomaterials (Parkatzidis et al., 2018).

Lately, lots of efforts have been made on pursuance of new approaches leading to further refinement of 3DLL manufacturing. Evidently, even simple and low-cost post-processing techniques may suggest a great potential. A possibility to apply ultra-fast 3D laser nano-lithography in conjunction with isotropic plasma etching and heat-treatment, leading to five fold scaling down, was already demonstrated (Seniutinas et al., 2018). Laser fabricated 3D structures, made from hybrid material SZ2080, containing silica and zirconia precursors. These precursors including the interconnecting oxygen species comprise $\sim 43 \mathrm{wt}$. \% of the resist in its sol-gel form. This part of resist after laser structuring, development and post-fabrication calcination - heating in air, starts to form silica and zirconia glass and crystalline phases. Near $1000^{\circ} \mathrm{C}$ such a procedure results in a structures comprised of a glass phase, and for $>1200^{\circ} \mathrm{C}$ a hybrid glass-ceramic phase emerges. Resolution in the final 3D structure is superior to that of the as-fabricated structures as homogeneous shrinking occurs down to $\sim 60 \%$ of initial value (Padolskyte et al., 2018).

The DLW 3D nano-lithography technique was successfully applied for the additive manufacturing of 3D metallic structures for electronic and mechanical applications (Blasco et al., 2016; Vyatskikh et al., 2018). Thus the 3D polymerization tool is becoming an equivalent to a colourful printer in a nano-scale where colours are represented by the diverse material spectrum. Also, an option of $3 \mathrm{D}$ nano-writing and selective erasing of the microstructures is possible via appropriate choice of materials and developers (Gräfe et al., 2018).

Not only the significantly decreased feature size but also completely different material properties obtained via calcination process are intriguing. Micro- and nanoscaled 3D ceramic structures, having higher refraction index (compared to polymers) and increased mechanical stability may open new possibilities in manufacturing of 
novel micro-optical components (Gissibl et al., 2016). No less important is that the process is highly repeatable and compatible with harsh industrial conditions. Further adjustment of chemical composition of hybrid photo-curable resins is possible and could be considered as a proper direction towards nano-printing of unique glassceramic or even crystalline materials (Gailevičius et al., 2018).

Currently, the state-of-the-art technique is right in between scientific research and industrial applications, the next generation burst mode lasers and dynamic tuning of polarization together with parallelization of the process will make it cost-efficient true 3D micro-/nano-fabrication tool. At the same time material properties will still have to be studied and optimized to match the high requirements in processing and functionality (Waller and von Freymann, 2016). Thus looking from the point of vies of occurring mechanisms during the laser processing, the topic will remain of great interest for the scientific community. At the same time industrial implementations will advance and benefit towards multi-scale and multi-material 3D micro-fabrication in both additive and subtractive manners (Wu et al., 2014) employing single fs-source (Tičkūnas et al., 2017) without caring too deep of the dominating physical/chemical mechanisms specifics.

\section{Acknowledgements}

Authors acknowledge the support by Lithuanian Science Council grants S-LAT17-2 "Optical 3D micro-/nanoprocessing of bioplastics (OPTIBIOFORM)". Special thanks are given to Prof. S. Juodkazis (Swinburne University of Technology, Melbourne) for its valuable insights leading to this work as it is.

\section{References}

Accardo A, Blatché MC, Courson R, Loubinoux I, Thibault C, Malaquin L, Vieu C (2017) Multiphoton direct laser writing and 3D imaging of polymeric freestanding architectures for cell colonization. Small 13(27):1700621, DOI 10.1002/smll.201700621

Baldacchini T, Snider S, Zadoyan R (2012) Two-photon polymerization with variable repetition rate bursts of femtosecond laser pulses. Opt Express 20(28):2989029899, DOI 10.1364/oe.20.029890

Beresna M, Kazansky PG (2010) Polarization diffraction grating produced by femtosecond laser nanostructuring in glass. Opt Lett 35(10):1662-1664, DOI 10.1364/ol.35.001662

Blasco E, Müller J, Müller P, Trouillet V, Schön M, Scherer T, Barner-Kowollik C, Wegener M (2016) Fabrication of conductive 3D gold-containing microstructures via direct laser writing. Adv Mater 28(18):3592-3595, DOI 10.1002/adma.201506126 
Buividas R, Rekštyte S, Malinauskas M, Juodkazis S (2013) Nano-groove and 3D fabrication by controlled avalanche using femtosecond laser pulses. Opt Mater Express 3(10):1674-1686, DOI 10.1364/ome.3.001674

Dietrich PI, Blaicher M, Reuter I, Billah M, Hoose T, Hofmann A, Caer C, Dangel R, Offrein B, Troppenz U, Moehrle M, Freude W, Koos C (2018) In situ 3D nanoprinting of free-form coupling elements for hybrid photonic integration. Nat Photonics 12:241-247, DOI 10.1038/s41566-018-0133-4

Do MT, Nguyen TTN, Li Q, Benisty H, Ledoux-Rak I, Lai ND (2013) Submicrometer 3D structures fabrication enabled by one-photon absorption direct laser writing. Opt Express 21(18):20964-20973, DOI 10.1364/oe.21.020964

Duan XM, Sun HB, Kaneko K, Kawata S (2004) Two-photon polymerization of metal ions doped acrylate monomers and oligomers for three-dimensional structure fabrication. Thin Solid Films 453-454:518-521, DOI 10.1016/j.tsf.2003.11.126

Fischer J, Wegener M (2011) Three-dimensional direct laser writing inspired by stimulated-emission-depletion microscopy [invited]. Opt Mater Express 1(4):614-624, DOI 10.1364/ome.1.000614

Fischer J, Mueller JB, Kaschke J, Wolf TJA, Unterreiner AN, Wegener M (2013) Three-dimensional multi-photon direct laser writing with variable repetition rate. Opt Express 21(22):26244-26260, DOI 10.1364/oe.21.026244

Gailevičius D, Padolskyte V, Mikoliūnaite L, Šakirzanovas S, Juodkazis S, Malinauskas M (2018) Additive-manufacturing of 3D glass-ceramics down to nanoscale resolution. Preprints 2018090412, DOI doi: 10.20944/preprints201809.0412.v1

Gamaly EG (2011) Femtosecond Laser-Matter Interaction: Theory, Experiments and Applications. Pan Stanford

Gissibl T, Thiele S, Herkommer A, Giessen H (2016) Sub-micrometre accurate freeform optics by three-dimensional printing on single-mode fibres. Nat Commun 7:11763, DOI 10.1038/ncomms11763

Gräfe D, Wickberg A, Zieger MM, Wegener M, Blasco E, Barner-Kowollik C (2018) Adding chemically selective subtraction to multi-material 3D additive manufacturing. Nat Commun 9(1):2788, DOI 10.1038/s41467-018-05234-0

Guo Q, Ghadiri R, Weigel T, Aumann A, Gurevich E, Esen C, Medenbach O, Cheng W, Chichkov B, Ostendorf A (2014) Comparison of in situ and ex situ methods for synthesis of two-photon polymerization polymer nanocomposites. Polymers 6(7):2037-2050, DOI 10.3390/polym6072037

Jonušauskas L, Lau $\mathrm{M}$, Gruber $\mathrm{P}$, Gökce $\mathrm{B}$, Barcikowski S, Malinauskas M, Ovsianikov A (2016) Plasmon assisted 3D microstructuring of gold nanoparticle-doped polymers. Nanotechnology 27(15):154001, DOI 10.1088/0957-4484/27/15/154001

Jonušauskas L, Gailevičius D, Mikoliūnaite L, Sakalauskas D, Šakirzanovas S, Juodkazis S, Malinauskas M (2017) Optically clear and resilient free-form $\mu$-optics 3D-printed via ultrafast laser lithography. Materials 10(1):12, DOI 10.3390/ma10010012

Jonušauskas L, Gailevičius D, Rekštyte S, Baldacchini T, Juodkazis S, Malinauskas M (2018) Mesoscale laser 3D printing. Preprints 2018100384, DOI 10.20944/preprints201810.0384.v1 
Juodkazis S, Mizeikis V, Seet KK, Miwa M, Misawa H (2005) Two-photon lithography of nanorods in SU-8 photoresist. Nanotechnology 16(6):846-849, DOI 10.1088/0957-4484/16/6/039

Li Z, Torgersen J, Ajami A, Mühleder S, Qin X, Husinsky W, Holnthoner W, Ovsianikov A, Stampflbe J, Liska R (2013) Initiation efficiency and cytotoxicity of novel water-soluble two-photon photoinitiators for direct 3D microfabrication of hydrogels. RSC Adv 3:15939-15946, DOI 10.1039/C3RA42918K

Malinauskas M, Žukauskas A, Bičkauskaite G, Gadonas R, Juodkazis S (2010) Mechanisms of three-dimensional structuring of photo-polymers by tightly focussed femtosecond laser pulses. Opt Express 18(10):10209-10221, DOI 10.1364/oe.18.010209

Malinauskas M, Danilevicius P, Juodkazis S (2011) Three-dimensional micro-/nanostructuring via direct write polymerization with picosecond laser pulses. Opt Express 19(6):5602-5610, DOI 10.1364/oe.19.005602

Malinauskas M, Žukauskas A, Hasegawa S, Hayasaki Y, Mizeikis V, Buividas R, Juodkazis S (2016) Ultrafast laser processing of materials: from science to industry. Light Sci Appl 5(8):e16133-e16133, DOI 10.1038/lsa.2016.133

Maruo S, Nakamura O, Kawata S (1997) Three-dimensional microfabrication with two-photon-absorbed photopolymerization. Opt Lett 22(2):132-134, DOI 10.1364/ol.22.000132

Mačiulaitis J, Deveikyte M, Rekštyte S, Bratchikov M, Darinskas A, Šimbelyte A, Daunoras G, Laurinavičiene A, Laurinavičius A, Gudas R, Malinauskas M, Mačiulaitis R (2015) Preclinical study of SZ2080 material 3D microstructured scaffolds for cartilage tissue engineering made by femtosecond direct laser writing lithography. Biofabrication 7(1):015015, DOI 10.1088/1758-5090/7/1/015015

Morikawa J, Orie A, Hashimoto T, Juodkazis S (2009) Thermal diffusivity in femtosecond-laser-structured micro-volumes of polymers. Appl Phys A 98(3):551-556, DOI 10.1007/s00339-009-5493-7

Mueller JB, Fischer J, Mange YJ, Nann T, Wegener M (2013) In-situ local temperature measurement during three-dimensional direct laser writing. Appl Phys Lett 103(12):123107, DOI 10.1063/1.4821556

Mueller JB, Fischer J, Wegener M (2016) Reaction mechanisms and in situ process diagnostics. In: Three-Dimensional Microfabrication Using Two-photon Polymerization, Elsevier, pp 82-101, DOI 10.1016/b978-0-323-35321-2.00005-4

Nguyen DTT, Tong QC, Ledoux-Rak I, Lai ND (2016) One-step fabrication of submicrostructures by low one-photon absorption direct laser writing technique with local thermal effect. J Appl Phys 119(1):013101, DOI 10.1063/1.4939294

Oakdale JS, Ye J, Smith WL, Biener J (2016) Post-print UV curing method for improving the mechanical properties of prototypes derived from two-photon lithography. Opt Express 24(24):27077-27086, DOI 10.1364/oe.24.027077

Ong BH, Yuan X, Tao S, Tjin SC (2006) Photothermally enabled lithography for refractive-index modulation in SU-8 photoresist. Opt Letters 31(10):1367-1369, DOI 10.1364/ol.31.001367

Padolskyte V, Gailevičius D, Jonušauskas L, Mikoliūnaite L, Katkus T, Gadonas R, Šakirzanovas S, Mizeikis V, Staliunas K, Juodkazis S, Malinauskas M (2018) 
3D opto-structuring of ceramics at nanoscale. Proc SPIE 10675:106750U, DOI 10.1117/12.2306883

Park SH, Lim TW, Yang DY, Kim RH, Lee KS (2006) Improvement of spatial resolution in nano-stereolithography using radical quencher. Macromol Res 14(5):559_ 564, DOI 10.1007/bf03218724

Parkatzidis K, Kabouraki E, Selimis A, Kaliva M, Ranella A, Farsari M, Vamvakaki M (2018) Initiator-free, multiphoton polymerization of gelatin methacrylamide. Macromol Mater Eng p 1800458, DOI 10.1002/mame.201800458

Peng Y, Jradi S, Yang X, Dupont M, Hamie F, Dinh XQ, Sun XW, Xu T, Bachelot R (2018) 3D photoluminescent nanostructures containing quantum dots fabricated by two-photon polymerization: Influence of quantum dots on the spatial resolution of laser writing. Adv Mater Technol p 1800522, DOI 10.1002/admt.201800522

Pikulin A, Bityurin N, Sokolov VI (2015) Model of diffusion-assisted direct laser writing by means of nanopolymerization in the presence of radical quencher. AIP Adv 5(12):127215, DOI 10.1063/1.4938512

Raman R, Bashir R (2015) Stereolithographic 3D bioprinting for biomedical applications. In: Essentials of 3D Biofabrication and Translation, Elsevier, pp 89-121, DOI 10.1016/b978-0-12-800972-7.00006-2

Rekštyte S (2016) The development and applications of the methods of direct laser writing using femtosecond light pulses in transparent cross-linkable materials. $\mathrm{PhD}$ thesis, Vilnius University

Rekštyte S, Jonušauskas L, Žukauskas A, Gervinskas G, Malinauskas M, Juodkazis S (2014a) Three-dimensional nanostructuring of polymer materials by controlled avalanche using femtosecond laser pulses. Proc SPIE 8972:897210, DOI 10.1117/12.2040971

Rekštyte S, Kaziulionyte E, Balčiūnas E, Kaškelyte D, Malinauskas M (2014b) Direct laser fabrication of composite material 3D microstructured scaffolds. J Laser Micro Nanoen 9(1):25-30, DOI 10.2961/jlmn.2014.01.0006

Rekštyte S, Jonavičius T, Gailevičius D, Malinauskas M, Mizeikis V, Gamaly EG, Juodkazis S (2016) Nanoscale precision of 3D polymerization via polarization control. Adv Opt Mater 4(8):1209-1214, DOI 10.1002/adom.201600155

Sakellari I, Kabouraki E, Gray D, Purlys V, Fotakis C, Pikulin A, Bityurin N, Vamvakaki M, Farsari M (2012) Diffusion-assisted high-resolution direct femtosecond laser writing. ACS Nano 6(3):2302-2311, DOI 10.1021/nn204454c

Seet KK, Juodkazis S, Jarutis V, Misawa H (2006) Feature-size reduction of photopolymerized structures by femtosecond optical curing of SU-8. Appl Phys Lett 89(2):024106, DOI 10.1063/1.2221499

Sekkat Z, Kawata S (2013) Laser nanofabrication in photoresists and azopolymers. Laser Photonics Rev 8(1):1-26, DOI 10.1002/lpor.201200081

Selimis A, Mironov V, Farsari M (2015) Direct laser writing: Principles and materials for scaffold 3D printing. Microelectron Eng 132:83-89, DOI 10.1016/j.mee.2014.10.001

Seniutinas G, Weber A, Padeste C, Sakellari I, Farsari M, David C (2018) Beyond $100 \mathrm{~nm}$ resolution in 3D laser lithography - post processing solutions. Microelectron Eng 191:25-31, DOI 10.1016/j.mee.2018.01.018 
Serbin J, Egbert A, Ostendorf A, Chichkov BN, Houbertz R, Domann G, Schulz J, Cronauer C, Fröhlich L, Popall M (2003) Femtosecond laser-induced twophoton polymerization of inorganic-organic hybrid materials for applications in photonics. Opt Lett 28(5):301-303, DOI 10.1364/ol.28.000301

Stankevičius E, Daugnoraite E, Selskis A, Juodkazis S, Račiukaitis G (2017) Photopolymerization differences by using nanosecond and picosecond laser pulses. Opt Express 25(5):4819-4830, DOI 10.1364/oe.25.004819

Sun HB, Maeda M, Takada K, Chon JWM, Gu M, Kawata S (2003) Experimental investigation of single voxels for laser nanofabrication via two-photon photopolymerization. Appl Phys Lett 83(5):819-821, DOI 10.1063/1.1598293

Suzuki T, Morikawa J, Hashimoto T, Buividas R, Gervinskas G, Paipulas D, Malinauskas M, Mizeikis V, Juodkazis S (2012) Thermal and optical properties of sol-gel and SU-8 resists. Proc SPIE 8249:8249OK, DOI 10.1117/12.907028

Takada K, Sun HB, Kawata S (2005) Improved spatial resolution and surface roughness in photopolymerization-based laser nanowriting. Appl Phys Lett 86(7):071122, DOI 10.1063/1.1864249

Takada K, Kaneko K, Li YD, Kawata S, Chen QD, Sun HB (2008) Temperature effects on pinpoint photopolymerization and polymerized micronanostructures. Appl Phys Lett 92(4):041902, DOI 10.1063/1.2834365

Tičkūnas T, Perrenoud M, Butkus S, Gadonas R, Rekštyte S, Malinauskas M, Paipulas D, Bellouard Y, Sirutkaitis V (2017) Combination of additive and subtractive laser 3D microprocessing in hybrid glass/polymer microsystems for chemical sensing applications. Opt Express 25(21):26280-26288, DOI 10.1364/OE.25.026280

Torgersen J, Ovsianikov A, Mironov V, Pucher N, Qin X, Li Z, Cicha K, Machacek T, Liska R, Jantsch V, Stampfl J (2012) Photo-sensitive hydrogels for threedimensional laser microfabrication in the presence of whole organisms. J Biomed Opt 17(10):105008, DOI 10.1117/1.jbo.17.10.105008

Ushiba S, Shoji S, Masui K, Kuray P, Kono J, Kawata S (2013) 3D microfabrication of single-wall carbon nanotube/polymer composites by two-photon polymerization lithography. Carbon 59:283-288, DOI 10.1016/j.carbon.2013.03.020

Varapnickas S, Baziulyte-Paulavičiene D, Šakirzanovas S, Malinauskas M (2018) Upconverting nanocrystals as luminescent temperature probes for localheating imaging during direct laser writing 3D nanolithography. Proc SPIE 10456:104564F, DOI 10.1117/12.2283224

Venkatakrishnan K, Tan B, Stanley P, Sivakumar NR (2002) The effect of polarization on ultrashort pulsed laser ablation of thin metal films. J Appl Phys 92(3):1604-1607, DOI 10.1063/1.1487453

Vetrone F, Naccache R, Zamarrón A, de la Fuente AJ, Sanz-Rodríguez F, Maestro LM, Rodriguez EM, Jaque D, Solé JG, Capobianco JA (2010) Temperature sensing using fluorescent nanothermometers. ACS Nano 4(6):3254-3258, DOI 10.1021/nn100244a

Vyatskikh A, Delalande S, Kudo A, Zhang X, Portela CM, Greer JR (2018) Additive manufacturing of 3D nano-architected metals. Nat Commun 9(1):593, DOI 10.1038/s41467-018-03071-9 
Žukauskas A, Batavičiūte G, Ščiuka M, Balevičius Z, Melninkaitis A, Malinauskas M (2015) Effect of the photoinitiator presence and exposure conditions on laserinduced damage threshold of ORMOSIL (SZ2080). Opt Mater 39:224-231, DOI 10.1016/j.optmat.2014.11.031

Waller EH, von Freymann G (2016) Spatio-temporal proximity characteristics in 3D $\mu$-printing via multi-photon absorption. Polymers 8(8):297, DOI 10.3390/polym8080297

Wickberg A, Mueller JB, Mange YJ, Fischer J, Nann T, Wegener M (2015) Threedimensional micro-printing of temperature sensors based on up-conversion luminescence. Appl Phys Lett 106(13):133103, DOI 10.1063/1.4916222

Wu D, Wu S, Xu L Jand Niu, Midorikawa K, Sugioka K (2014) Hybrid femtosecond laser microfabrication to achieve true 3D glass/polymer composite biochips with multiscale features and high performance: the concept of ship-in-a-bottle biochip. Laser Photon Rev 8(3):458-467, DOI 10.1002/lpor.201400005

Yagci Y, Jockusch S, Turro NJ (2010) Photoinitiated polymerization: Advances, challenges, and opportunities. Macromolecules 43(15):6245-6260, DOI $10.1021 / \mathrm{ma1} 007545$ 


\section{Index}

3D laser lithography process, 3

3D micro-structure, 4

micro-optical component, 12, 25

photonic crystal, 10, 21, 23

scaffold, 10-12, 21

absorption

linear, 4, 12, 16

multi-photon, 6, 7, 9, 20

non-linear, $3,5,11,15$

two-photon, 4, 7, 9, 10

avalanche ionization, $6,8,9,12,15$

calcination, 24

degree of cross-linking, 22

direct laser writing, 2, 4

direct laser writing setup, 5

intensity distribution, 4

LIDT, 11, 12

nano-lithography, 9, 19, 24

nanoparticles, $3,12,13,24$

numerical aperture, 3,18

over-exposition, 11,14

photo-initiator, 7, 10 polarization control, 16, 21, 25

polymerization threshold, 6

post-processing, 24

pre-polymer, 3, 12, 18

GelMa, 12

OrmoClear, 11

OrmoComp, 17, 19

PDMS, 9, 12

PEG-DA, 9, 12, 17

PETA, 14, 16, 17, 19

SCR500, 16, 22

SU-8, 15

SZ2080, 9, 12, 15, 19, 23

processing window, 11

pulse duration, $2,6,7,12,22$

femtosecond, 2, 7, 9, 14, 15

nanosecond, 2, 7

picosecond, 15

radical density, 7

radical quencher, 22

structuring resolution, 5, 6, 12, 13, 15, 16, 22, 24

temperature effects, $13,14,16$

thermal accumulation, 15, 16

voxel, 4 